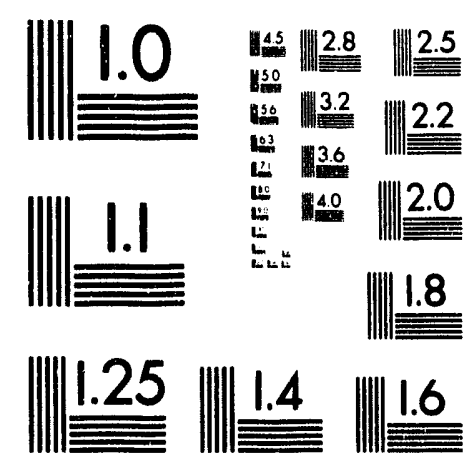



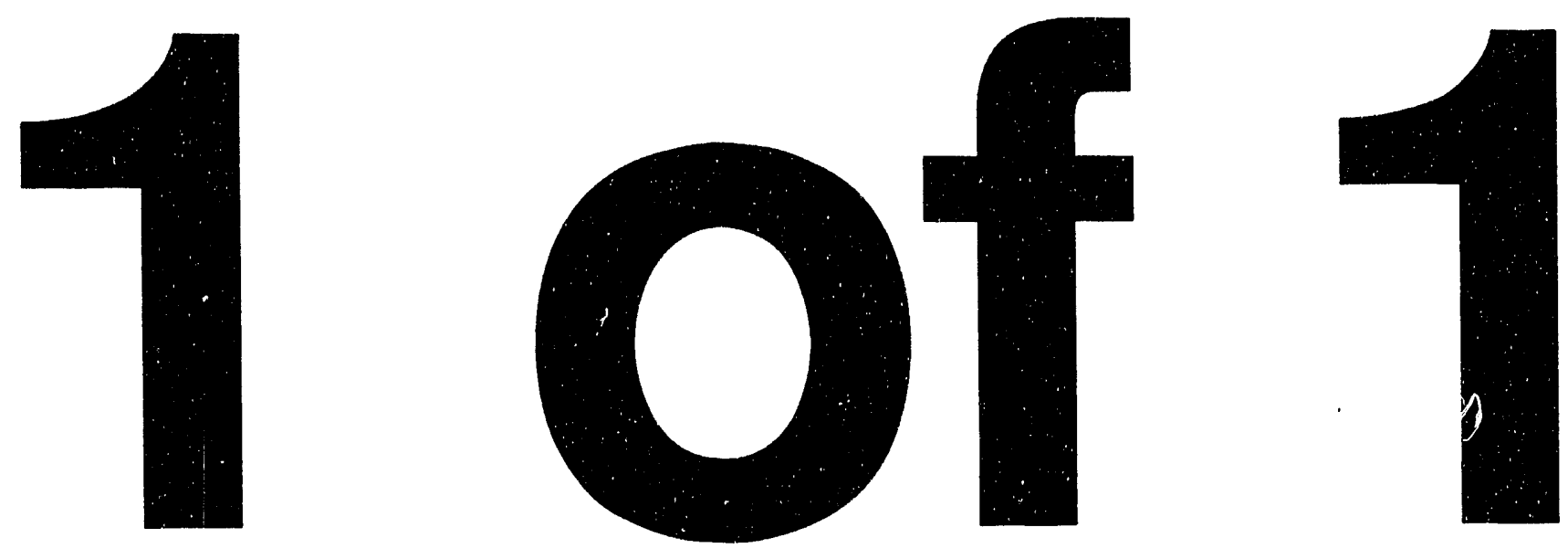


\section{Continuum and Micromechanics Treatment of Constraint in Fracture}

Manuscript Completed: February 1993

Date Published: July 1993

\section{Prepared by}

R. H. Dodds, Jr., University of Illinois

C. F. Shih, Brown University

T. L. Anderson, Texas A\&M University

University of Illinois at Urbana-Champaign

Department of Civil Engineering, MC-250

205 North Mathews Avenue

Urbana, Il 61801-2352

Brown University

Division of Engineering

Providence, RI 02912

Texas A\&M University

Department of Mechanical Engineering

College Station, TX 77843

Under Contract to:

Naval Surface Warfare Center

Annapolis Detachment, Carderock Division

Code 2814

Annapolis, MD 21402-5067

Prepared for

Division of Engineering

Office of Nuclear Regulatory Research

U.S. Nuclear Regulatory Commission

Washington, DC 20555-0001

NRC FIN B6290

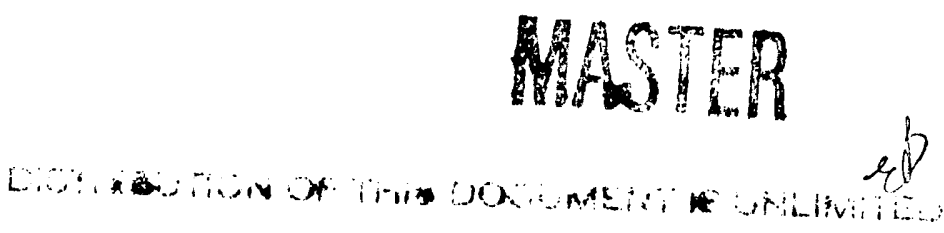




\section{ABSTRACT}

Two complementary methodologies are described to quantify the effects of crack-tip stress triaxiality (constraint) on the macroscopic measures of elastic-plastic fracture toughness, $J$ and Crack-Tip Opening Displacement (CTOD). In the continuum mechanics methodology, two parameters, $J$ and $Q$, suffice to characterize the full range of near-tip environments at the onset of fracture. $J$ sets the size scale of the zone of high stresses and large deformations while $Q$ scales the near-tip stress level relative to a high triaxiality reference stress state. Full-field finite element calculations show that the $J-Q$ field dominates over physically significant size scales, i.e., it describes the environment in which brittle and ductile failure mechanisms are active. The material's fracture resistance is characterized by a toughness locus, $J_{c}(Q)$, which defines the sequence of $J-Q$ values at fracture determined by experiment from high constraint conditions $(Q \approx 0)$ to low constraint conditions $(Q<0)$.

To reduce experimental effort needed to construct a $J-Q$ toughness locus, a micromechanics methodology is described which predicts the toughness locus using crack-tip stress fields and critical $J$-values from a few fracture toughness tests. A robust micromechanics model for cleavage fracture has evolved from the observations of a strong, spatial self-similarity of crack-tip principal stresses under increased loading and across different fracture specimens. While the spatial variation remains self-similar, the magnitudes of principal stresses vary dramatically as crack-tip constraint evolves under loading. The micromechanics model employs the volume of material bounded within principal stress contours at fracture to correlate $J_{c}$ values for different specimens and loading modes. The $J-Q$ description of the crack-tip stress fields predicts the similarity of principal stress contours as constraint evolves under loading. For an applied el-value, the size, but not the shape, of principal stress contours is altered by the near-tip, uniform hydrostatic stress states of adjustable magnitude characterized by $Q$. These observations imply that values specified for metallurgical parameters in the micromechanics model, such as the critical fracture stress and the distance to the critical particle, have only a weak influence on the relative variation of fracture toughness, $J_{c}$, with constraint for a given material and temperature.

This report explores the fundamental concepts of the $J-Q$ description of crack-tip fields, the fracture toughness locus and micromechanics approaches to predict the variability of macroscopic fracture toughness with constraint under elastic-plastic conditions. While these concepts derived from plane-strain considerations, initial applications in fully 3-D geometries are very promising. Computational results are presented for a surface cracked plate containing a 6:1 semi-elliptical, $a=t / 4$ flaw subjected to remote uniaxial and biaxial tension. Crack-tip stress fields consistent with the $J-Q$ theory are demonstrated to exist at each location along the crack front. The micromechanics model employs the $J-Q$ description of crack-front stresses to interpret fracture toughness values measured on laboratory specimens for fracture assessment of the surface cracked plate. The computational results suggest only a minor effect of the biaxial loading on the crack tip stress fields and, consequently, on the propensity for fracture relative to the uniaxial loading. 


\section{Contents}

Section No.

Page

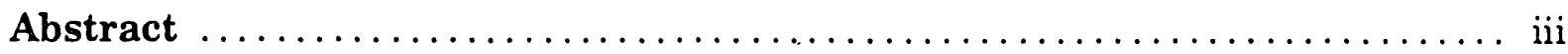

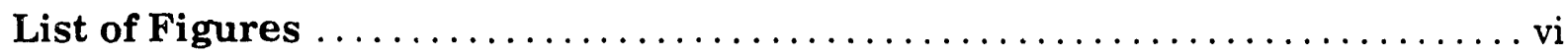

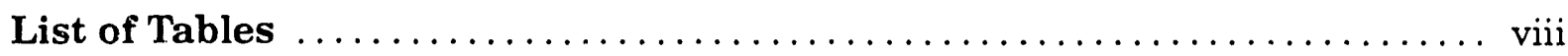

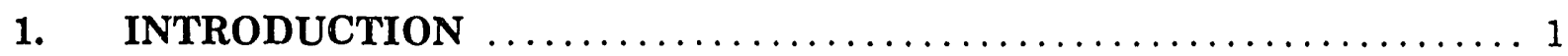

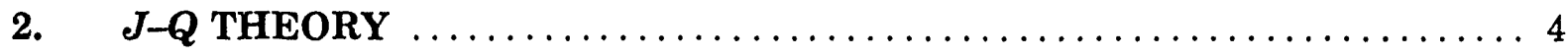

$2.1 Q$-Family of Fields-MBL Formulation $\ldots \ldots \ldots \ldots \ldots \ldots \ldots \ldots$

2.2 Difference Field and Near-Tip Stress Triaxiality $\ldots \ldots \ldots \ldots \ldots \ldots .5$

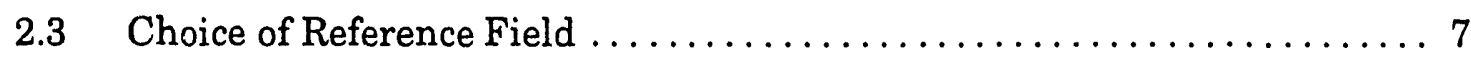

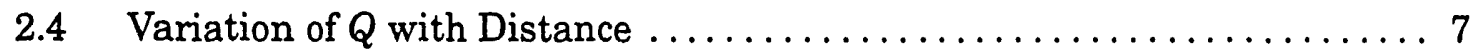

2.5 Simplified Forms for Engineering Applications $\ldots \ldots \ldots \ldots \ldots \ldots \ldots 8$

2.6 Difference Field and Higher-Order Terms of the Asymptotic Series .... 9

$2.7 J-Q$ Material Toughness Locus .......................... 10

3. MICROMECHANICAL CONSTRAINT CORRECTIONS $\ldots \ldots \ldots \ldots \ldots 12$

3.1 Transgranular Cleavage Mechanism $\ldots \ldots \ldots \ldots \ldots \ldots \ldots \ldots \ldots . \ldots \ldots$

3.2 Development of the Constraint Corrections $\ldots \ldots \ldots \ldots \ldots \ldots \ldots 13$

3.3 Application of Constraint Corrections in Fracture Testing $\ldots \ldots \ldots \ldots 18$

3.4 Engineering Use of $J-Q$ Fields in the Micromechanics Model ........ 19

4. SURFACE CRACKS UNDER UNIAXIAL AND BIAXIAL LOADING . . . . 23

4.1 Part-Through Surface Crack Model $\ldots \ldots \ldots \ldots \ldots \ldots \ldots \ldots \ldots . \ldots 23$

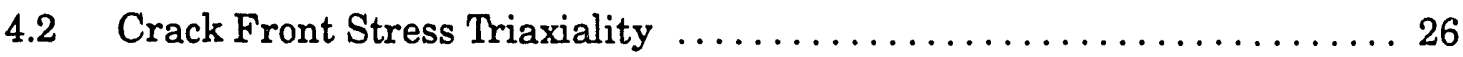

4.3 Matching Structural and Test Specimen Constraint ............. 29

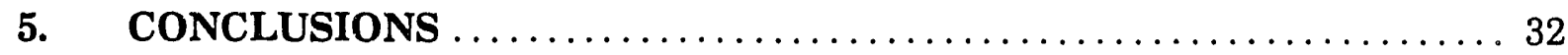

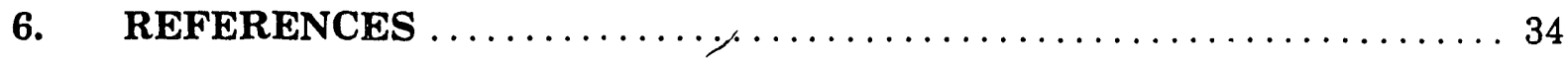




\section{LIST OF FIGURES}

Figure No.

Page

2.1 Definition of the Modified Boundary Layer (MBL) problem $\ldots \ldots \ldots \ldots \ldots \ldots \ldots$

2.2 Plane strain reference fields for $n=10, E / \sigma_{0}=500, v=0.3$. Reference fields for several $n$ values are tabulated in Table 2.1. The material stress-strain curve has the form

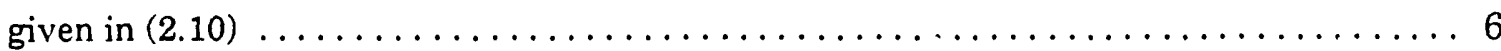

2.3 Application of the $J-Q$ methodology in fracture assessments. (a) Laboratory testing of specimens with varying constraint to measure the material's fracture resistance. Circles indicate anticipated scatter which define upper-lower bounds. (b) Evaluation of structural flaws using measured toughness locus and predicted $J-Q$ response for two structural configurations. Cleavage fracture is predicted for Structure A;

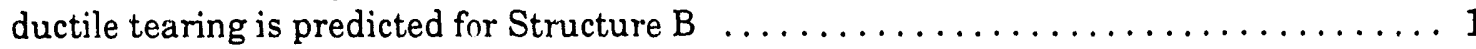

3.1 Comparison of a maximum principal stress contour for $\mathrm{SSY}_{\mathrm{Q}=0}$ with those for an $a / W=0.15, n=10 \mathrm{SE}(\mathrm{B})$. $\mathrm{SE}(\mathrm{B})$ contours decrease in size with increasing

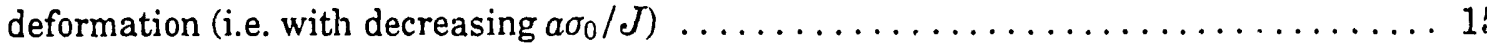

3.2 Areas within principal stress contours for an $a / W=0.15, n=10 \mathrm{SE}(\mathrm{B})$. Values are normalized by area within contour for $\mathrm{SSY}_{\mathrm{Q}=0}$ at same $J$-value $\ldots \ldots \ldots \ldots \ldots \ldots$

3.3 Influence of specified critical stress on the micromechanics prediction of fracture toughness variation with constraint for an $a / W=0.15, n=10 \mathrm{SE}(\mathrm{B}) \ldots \ldots \ldots \ldots \ldots \ldots$

3.4 Micromechanics prediction of $a / W$ effects on cleavage fracture toughness for an $n=5$,

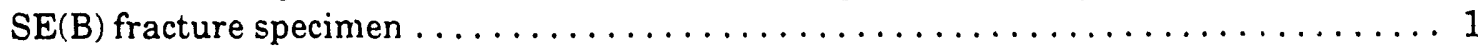

3.5 (a) Effect of initial crack depth on cleavage fracture toughness in a mild steel (Sumpter and Forbes [10]; (b) $J_{0}$ values (specimen size independent fracture toughness) calculated from experimental $J_{c}$ data using the micromechanics cor straint

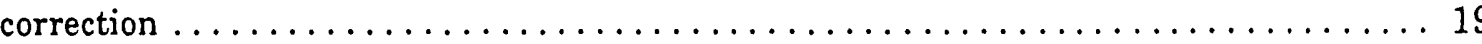

3.6 Opening stress on the crack plane normalized by $\mathrm{SSY}_{\mathrm{Q}=0}$ at same $J$-applied

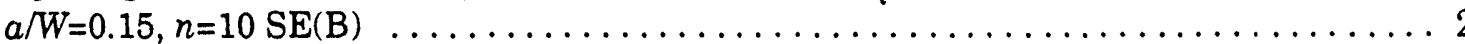

3.7 Variation of fracture toughness predicted by the micromechanics model using crack-plan€ stress shown in Fig. 3.6. $a / W=0.15, n=10 \mathrm{SE}(\mathrm{B}) \ldots \ldots \ldots \ldots \ldots \ldots \ldots$

3.8 Comparison of $J_{0}$ values determined using the crack-plane stress from $J-Q$ and stressed volume techniques for an $a / W=0.15 n=10 \mathrm{SE}(\mathrm{B})$

4.1 Finite-element model for investigation of constraint in surface cracked plate subjected to uniaxial and biaxial remote tension loadings

4.2 Global load-displacement (Crack Mouth Opening Displacement, CMOD) response for surface cracked plate

$4.3 \mathrm{~J}$-integral values at point of maximum crack depth for uniaxial and biaxial tension loading of surface cracked plate model $\ldots \ldots \ldots \ldots \ldots \ldots \ldots \ldots \ldots \ldots \ldots$

$4.4 J$-integral along crack front at SSY and LSY load levels normalized by corresponding

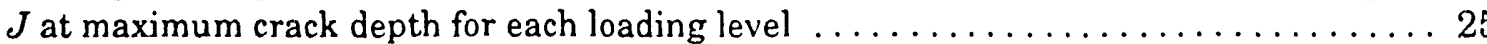


4.5 Evolution of stress triaxiality in surface cracked plate ahead of crack tip at $\phi=90^{\circ}$ and $17^{\circ}$

4.6 Comparison of $Q$-values computed using opening-mode stress (2.9) and the mean stress (2.14): (a) $\mathrm{SE}(\mathrm{B})$ specimen with $a / W=0.15, n=10$;

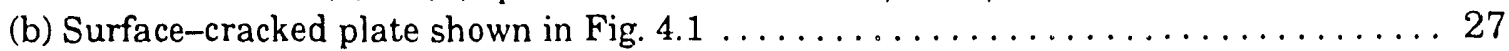

4.7 Evolution of stress triaxiality in surface-cracked plate with increasing local deformation, as measured by $J_{l o c a l} / a \sigma_{0}$, along radial lines emanating from different points on the crack front. $Q$ is evaluated at $r /\left(J_{l o c a l} / \sigma_{0}\right)=2$. Both geometries are loaded to the same level $\sigma \infty / \sigma_{0}=1.1 \ldots \ldots \ldots \ldots \ldots \ldots \ldots \ldots \ldots \ldots \ldots \ldots \ldots$

4.8 Application of $J-Q$ methodology to select $a / W$ ratio in $\mathrm{SE}(\mathrm{B})$ specimen for testing that matches stress triaxiality in structural configuration represented by a surface cracked plate loaded in uniaxial or biaxial tension $\ldots \ldots \ldots \ldots \ldots \ldots \ldots$

4.9 Application of micromechanics methodology to select $a / W$ ratio in $\mathrm{SE}(\mathrm{B})$ specimen for testing that matches effective driving force for cleavage fracture in structural configuration represented by a surface cracked plate loading in uniaxial or biaxial tension 


\section{LIST OF TABLES}

Table No.

Page

2.1 Reference stresses, $\sigma_{\theta \theta} / \sigma_{0}$, for MBL problem, $T / \sigma_{0}=0 \ldots \ldots \ldots \ldots \ldots \ldots$

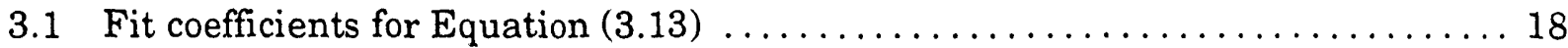

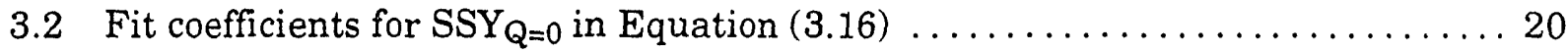

4.1 Predicted Loads at Fracture for Surface-Cracked Plate . . . . . . . . . . . 31 


\section{Continuum and Micromechanics Treatment of Constraint in Fracture}

\section{INTRODUCTION}

Two fundamental concepts underlie both linear-elastic fracture mechanics and elasticplastic fracture mechanics: [1] the relevant crack-tip singularity dominates over microstructurally significant size scales and [2] the parameter $K_{I}$, or $J$, uniquely scales the amplitude of the near-tip fields. In an actual structure, the crack-tip field must be perturbed by the external boundary and the loading distribution (from a far), and by the zone of inelasticity and small-scale heterogeneities, e.g., grains, microcracks, crack face roughness (from within). However, when the zone of inelasticity and small-scale heterogeneities remains small compared to the external geometry, the asymptotic field is approximately unperturbed in an annulus which is larger than the zone of inelasticity and small-scale heterogeneities, but much smaller than the external geometry. Strain-stress fields in such an annulus are determined completely by the singularity solution (see review article by Hutchinson [1]). Under this condition, the effects of remote loading and external boundaries are communicated to the crack tip through $K_{I}$, or $J$, alone. Moreover, when linear elasticity prevails at the macro-scale, then boundary loading as well as traction free boundaries exert their influence on the near-tip field through $K_{I}$ alone, with no effect on the actual distribution. Similarly, the plastic fields for well-contained yielding sense external boundaries and loading only through $J[2,3,4]$. However, for large-scale yielding in finite bodies, the relationship between the scaling parameter, $J$, and the near-tip fields loses the one-to-one correspondence $[5,6,7]$ This loss of uniqueness, often termed loss of constraint, produces the increases in fracture toughness observed for tension geometries and for shallow notch bend specimens. The mismatch of constraint conditions at the crack tip apparently plays a dominant role in the often disappointing correlation between fracture specimen behavior $[\mathrm{C}(\mathrm{T})$, $\mathrm{SE}(\mathrm{B})$ ] and the behavior observed in large-scale, tension loaded tests.

Constraint effects are most pronounced for low-to-medium strength structural steels (and their weldments) operating in the ductile-to-brittle transition region where unstable fracture occurs by the micromechanism of transgranular cleavage. Many nuclear, civil and marine structures operate in the transition region over significant portions of their lifetimes. Unlike the more ductile mechanism of slow stable tearing, cleavage fractures most often trigger catastrophic failure of even highly redundant structural systems. Extensive experimental studies (see, for example [8-12]) have readily demonstrated the much greater sensitivity of cleavage fracture toughness, $J_{c}$, to constraint than is observed for ductile initiation toughness, $J_{I c}$, and for ductile crack growth resistance $J-\Delta a$.

In the past three years, new approaches have appeared to quantify constraint and to predict the effects of constraint changes on macroscopic (engineering) fracture toughness characterized by $J$ and the crack tip opening displacement, CTOD or $\delta$. Very detailed, elastic-plastic finite element analyses provide correlations of crack-tip stress fields over distances $r \leq 1-8 \delta$ with loading level ( $J)$, loading mode (tension $v s$. bending), specimen geometry and strain hardening. Such computations stimulated development of the $J-Q$ continuum mechanics framework [13-19] to describe the near-tip fields under very general conditions of loading in finite bodies. Within this framework, the $J$-integral sets the scale of deformation at the crack tip (i.e., the CTOD) while the hydrostatic stress parameter, $Q$, quan- 
tifies the level of stress triaxiality over distances $r \leq 1-8 \delta$ ahead of the tip in which the microseparation processes occur. Under increased loading, each fracture specimen for a specific material/temperature fo!lows a characteristic $J-Q$ driving force curve which defines the evolution of crack-tip deformation and constraint. Specimens fracture at critical $J$-values which depend on $Q$ (critical $J$-values are determined by laboratory testing, the corresponding $Q$-values at fracture are determined from analysis). By testing fracture specimens that exhibit a wide-range of constraint conditions (e.g. shallow-to-deep notch $\mathrm{SE}(\mathrm{B})$ specimens), the toughness locus for the material is constructed, i.e., the curve connecting all critical Jus. $Q$ points.

The level of stress triaxiality, quantified conveniently by $Q$, plays a dominant role in the competition between fracture initiation by cleavage and ductile tearing for ferritic materials in the ductile-to-brittle transition region. Cleavage fracture is controlled by critical levels of the hoop stress (opening) acting over microstructurally significant distances ahead of the crack tip [20,21]. Ductile tearing is controlled by several competing processes, including growth of a dominant void and coalesescence with the crack tip, interaction of many growing voids leading to localization of plastic flow, and zigzag mode of ductile tearing $[22,23]$. Each of these mechanisms is dependent to some extent on the mean stress. Since $Q$ quantifies both the hoop stress and the mean stress over the operative length scales for both cleavage and ductile micromechanisms, it provides a common parameter to interpret fracture initiation in terms of a single toughness locus.

Another two-parameter approach also receiving considerable attention utilizes $J$ and the elastic $T$-stress [7,24-29]. These studies propose to correlate crack-tip stress triaxiality in contained and fully-yielded cracked bodies using the the elastic $T$-stress. The $J-T$ and $J-Q$ approaches are equivalent under well-contained yielding conditions [14-16]. However, under fully-yielded conditions the $T$-stress becomes undefined; moreover, $T$ is proportional to $K_{I}$ and near limit load $K_{I}$ (and thus $T$ ) approaches a saturation value independent of additional plastic deformation. In contrast, the $Q$-parameter continues to evolve over the entire range of plastic yielding. Numerical studies have shown that the $J-T$ approach overestimates the actual stress triaxiality for some geometries and underestimates it in other cases so that there is not a consistent trend [16,30]. An extensive study of the limits of applicability of the $T$-stress as a correlator of near-tip stress triaxiality can be found in [30]. Readers are referred to the publications [7,24-29] and references therein for details of the $J-T$ approach. This paper focuses on continuum and micromechanics approaches believed to have broader applicability.

The $J-Q$ approach may become prohibitively expensive as the number of specimens and temperatures of interest increases. To reduce the cost, a micromechanics model for cleavage fracture is introduced to predict the toughness locus using the finite element stress fields and the $J_{C}$ values from a few fracture toughness tests. Recent developments $[31,32]$ in the formulation of a robust micromechanics model focus on the observation of a strong, spatial self-similarity of crack-tip principal stresses under increased loading and across different fracture specimens. While the spatial variation remains self-similar, the magnitudes of principal stresses vary dramatically as crack-tip constraint evolves under loading. The micromechanics model employs the volume of material bounded within principal stress contours at fracture to correlate $J_{c}$ values for different specimens and loading modes. The similarity of principal stress contours as constraint evolves under loading is entirely consistent with the $J-Q$ description of the crack-tip stress fields. For an applied $J$-value, the sizc, but not the shape, of principal stress contours is altered by the near-tip, uniform hydrostatic stress states of adjustable magnitude characterized by $Q$. These observations imply that 
values specified for metallurgical parameters in the micromechanics model, such as the critical fracture stress and the distance to the critical particle, have only a weak influence on the relative variation of fracture toughness, $J_{c}$, with constraint for a given material and temperature.

This paper explores the fundamental concepts of the $J-Q$ description of crack-tip fields, the fracture toughness locus and micromechanics approaches to predict the variability of macroscopic fracture toughness with constraint under elastic-plastic conditions. While these concepts derived from plane-strain considerations, initial applications in fully 3-D geometries are very promising. Computational results are presented for a surface cracked plate containing a 6:1 semi-elliptical, $a=t / 4$ flaw subjected to remote uniaxial and biaxial tension. Crack-tip stress fields consistent with the $J-Q$ theory are demonstrated to exist at each location along the crack front. The micromechanics model employs the $J-Q$ description of crack-front stresses to interpret fracture toughness values measured on laboratory specimens for fracture assessment of the surface cracked plate. The computational results suggest only a minor effect of the biaxial loading on the crack tip stress fields and, consequently, on the propensity for fracture relative to the uniaxial loading. 


\section{J-Q THEORY}

Consider a cracked body of characteristic dimension $L$ loaded remotely by a stress denoted $\sigma \infty$. The scale of crack-tip deformation is measured by $J / \sigma_{0}$ where $\sigma_{0}$ is the material's tensile yield stress $\left(\delta \propto J / \sigma_{0}\right)$. At a sufficiently low load, $L \gg J / \sigma_{0}$ and it can be shown from dimensional grounds that all near-tip fields are members of a single family of crack-tip fields. Each member field is characterized by its level of deformation as measured by $J / \sigma_{0}$ and by its level of crack tip stress triaxiality as measured by $Q$, which also identifies that field as a particular member of the family. For example, the self-similar solution of Rice and Johnson [33] and McMeeking [34] (as well as the HRR field [3,4]) is the $Q \equiv 0$ member field. The $Q$-family of fields provides the proper characterizing parameter for the full range of near-tip stress states.

In the following discussion, attention is directed to the prospective fracture region ahead of the crack tip on the scale of several crack opening displacements, $\delta$, representing the environment in which the failure mechanisms are active.

\subsection{Q-Family of Fields-MBL Formulation}

The Q-family of fields is constructed using a modified boundary layer (MBL) formulation in which the remote tractions are given by the first two terms of the small-displacementgradient linear elastic solution (Williams [35]),

$$
\sigma_{i j}=\frac{K_{I}}{\sqrt{2 \pi r}} \tilde{f}_{i j}(\theta)+T \delta_{1 i} \delta_{1 j}
$$

Here $r$ and $\theta$ are polar coordinates centered at the crack tip with $\theta=0$ corresponding to a line ahead of the crack is shown in the insert in Fig. 2.1. Cartesian coordinates, $X$ and $Y$ with the $X$-axis running directly ahead of the crack, are used when it is convenient. Within the MBL formulation,

$$
J=\frac{1-v^{2}}{E} K_{I}^{2}
$$

under plane strain conditions, where $J$ is Rice's $J$-integral [1], $E$ is Young's modulus and $v$ is Poisson's ratio.

Fields of different crack tip stress triaxialities can be induced by applying different combinations of $K$ and $T$. From dimensional considerations, these fields can be organized into a family of crack tip fields parameterized by $T / \sigma_{0}$ :

$$
\sigma_{i j}=\sigma_{0} \bar{f}_{i j}\left(\frac{r}{J / \sigma_{0}}, \theta ; T / \sigma_{0}\right) \text {. }
$$

That is, the load parameter $T / \sigma_{0}$ provides a convenient means to investigate and parameterize specimen geometry effects on near-tip stress triaxiality under conditions of wellcontained yielding. Such studies have been carried out by Betegon and Hancock [24], Bilby et al. [36] and Harlin and Willis [37]. Nevertheless, the result in (2.3) cannot have general applicability since the elastic solution (2.1), upon which the $T$-stress is defined, is an asymptotic condition which is increasingly violated as plastic flow progresses beyond wellcontained yielding.

Recognizing the above limitation, O'Dowd and Shih [13,14], referred to as OS, identified members of the family of fields by the parameter $Q$ which arises naturally in the plasticity analysis. OS write: 


$$
\sigma_{i j}=\sigma_{0} f_{i j}\left(\frac{r}{J / \sigma_{0}}, \theta ; Q\right), \varepsilon_{i j}=\varepsilon_{0} f_{i j}\left(\frac{r}{J / \sigma_{0}}, \theta ; Q\right), u_{i}=\frac{J}{\sigma_{0}} h_{i}\left(\frac{r}{J / \sigma_{0}}, \theta ; Q\right) .
$$

The additional dependence of $f_{i j}, g_{i j}$ and $h_{i}$ on dimensionless combinations of material parameters is understood. The form in (2.4) constitutes a one-parameter family of self-similar solutions, or in short a $Q$-family of solutions. The annular zone over which (2.4) accurately quantifies the actual field is called the $J-Q$ annulus.

\subsection{Difference Field and Near-Tip Stress Triaxiality}

Using the modified boundary layer formulation, and considering a piecewise, power-law hardening material, OS generated the full range of small scale yielding, plane strain solutions, designated by $\left(\sigma_{i j}\right)_{S S Y}$. OS considered the difference field defined by

$$
\Delta \sigma_{i j}=\left(\sigma_{i j}\right)_{\mathrm{SSY}}-\left(\sigma_{i j}\right)_{\mathrm{HRR}}
$$

where $\left(\sigma_{i j}\right)_{\mathrm{HRR}}$ is the HRR field. They systematically investigated the difference field within the forward sector $|\theta|<\pi / 2$ of the annulus $J / \sigma_{0}<r<5 J / \sigma_{0}$, since this zone encompasses the microstructurally significant length scales for both brittle and ductile fracture[20]. Rernarkably, the difference field in the forward sector displayed minimal dependence on $r$. Noting this behavior, OS expressed the difference field within the forward sector in the form

$$
\Delta \sigma_{i j}=Q \sigma_{0} \hat{\sigma}_{i j}(\theta)
$$

where the angular funcions $\hat{\sigma}_{i j}$ are normalized by requiring $\sigma_{i j}(\theta=0)$ to equal unity. Moreover, the angular functions within the forward sectur exhibit these features: $\sigma_{r r} \approx \sigma_{\theta \theta} \approx$ constan $i$ and $\left|\sigma_{r \theta}\right| \ll\left|\sigma_{\theta \theta}\right|$ (see Figs. 3, 4, and 5 in [13]).

Thus the difference field within the sector $|\theta|<\pi / 2$ and $J / \sigma_{0}<r<5 J / \sigma_{0}$, correspond effectively to a spatially uniform hydrostatic stress state of adjustable magnitude, i.e. $\Delta \sigma_{i j}=Q \sigma_{0} \delta_{i j}$. Therefore $Q$ defined by

$$
Q \equiv \frac{\sigma_{\theta \theta}-\left(\sigma_{\theta \theta}\right)_{\mathrm{HRR}}}{\sigma_{0}} \text { at } \theta=0, \quad r=2 . J / \sigma_{0}
$$

is a natural measure of near-tip stress triaxiality, or crack tip constraint, relative to a high triaxiality reference stress state. For definiteness we have evaluated $Q$ at $r=2 J / \sigma_{0}$, however we note that $Q$ is efffectively independent of distance. In words, $Q$ is the difference between the actual hoop stress and the corresponding HRR stress component at $r=2 J / \sigma_{0}$, the difference being normalized by $\sigma_{0}$. The distance chosen for the definition of $Q$ lies just outside the finite strain blunting zone. It is preferable that $Q$ be defined at a distance which is some multiple of the crack tip opening displacement; the present definition suffices for our purposes.

OS also considered the difference field whereby the reference solution is the standard small scale yielding solution, $\left(\sigma_{i j}\right)_{\mathrm{SSY} ; \mathrm{T}=0}$ which is driven by $K_{I}$ alone, i.e.,

$$
\Delta \sigma_{i j}=\left(\sigma_{i j}\right)_{\mathrm{SSY}}-\left(\sigma_{i j}\right)_{\mathrm{SSY} ; \mathrm{T}=0}
$$

In this case the difference field in the forward sector matches a spatially uniform hydrostatie stress state even more closely. Thus an alternative definition of $Q$ is

$$
Q \equiv \frac{\sigma_{\theta \theta}-\left(\sigma_{\theta \theta}\right)_{\mathrm{SSY} ; \mathrm{T}=0}}{\sigma_{0}} \text { at } \theta=0, \quad r=2 J / \sigma_{0} .
$$

Representative stress distributions of the $Q$-family of fields can be found in $[13,14]$. 


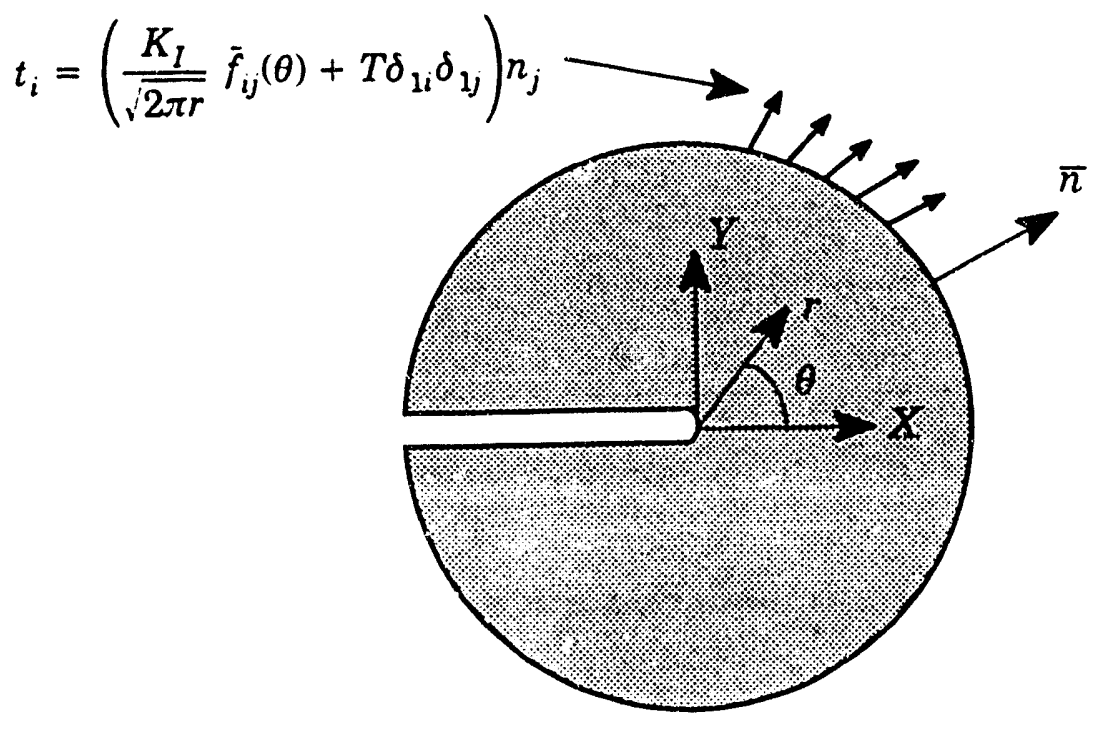

Figure 2.1 Definition of the Modified Boundary Layer (MBL) problem.

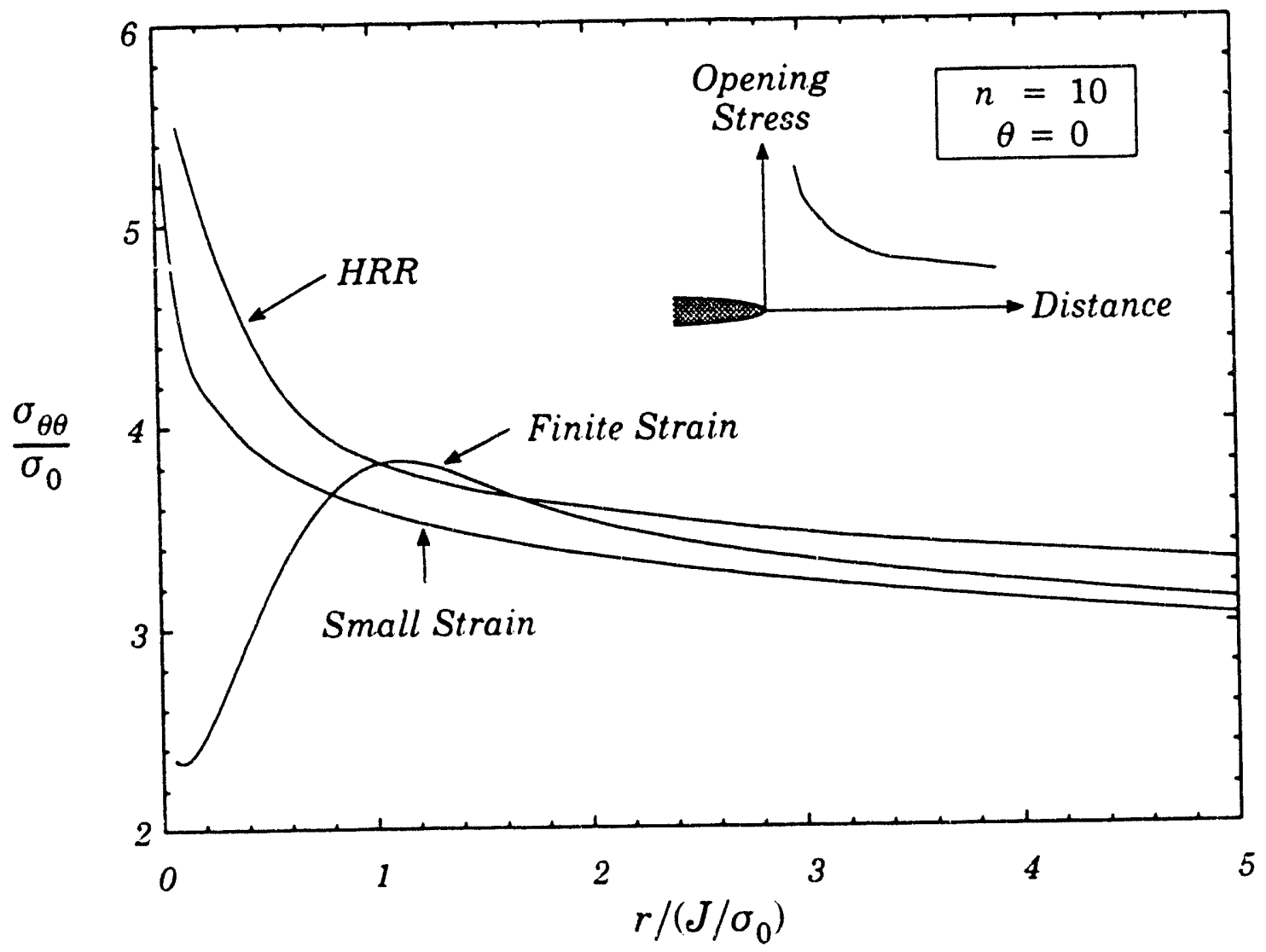

Figure 2.2 Plane st rain reference fields for $n=10, E ; \sigma_{(i}=500, v=0.3$. Reference fields for several $n$ values are tabulated in Table 2.1. The material stress-strain curve has the form given in (2.10). 


\subsection{Choice of Reference Field}

The value of $Q$ is slightly affected by the choice of reference field. Thus a small increment (or decrement) must be applied to the $Q$-values if the reference field is changed from $\left(\sigma_{\theta \theta}\right)_{\mathrm{HRR}}$ to $\left(\sigma_{\theta \theta}\right)_{\mathrm{SSY}} \mathrm{T}=0$, or vice versa. The reference field distributions according to the HRR singularity and the small scale yielding solutions for small strain and finite strain are given in Table 2.1. The material's uniaxial stress-strain response is represented by an elastic power-law model having the form

$$
\varepsilon=\left\{\begin{array}{ll}
\sigma / E & \text { if } \sigma \leq \sigma_{0} \\
\varepsilon_{0}\left(\sigma / \sigma_{0}\right)^{n} & \text { if } \sigma>\sigma_{0}
\end{array} ; \varepsilon_{0}=\sigma_{0} / E\right.
$$

with values of $\mathrm{E} / \sigma_{0}=500, v=0.3$ adopted in the computations. Figure 2.2 shows typical reference fields determined from the MBL formulation with $T=0$.

In practice it really does not matter whether we use $\left(\sigma_{\theta \theta}\right)_{\mathrm{HRR}}(2.7)$, or $\left(\sigma_{\theta \theta}\right)_{\mathrm{SSY} ; \mathrm{T}=0}(2.9)$, for the definition of $Q$ so long as it is applied consistently. In other words, the evaluation and tabulation of $Q$ solutions for test specimens, the determination of the toughness locus from test data, and subsequent applications of such data to predict fracture in structural components should be based on the same reference field. Nevertheless, use of (2.9) can extend the range of applicability of the $J-Q$ approach and is preferable when it is desired to assess the spatial extent of the $J-Q$ annulus. A parameter which can ascertain the robustness of the $J-Q$ field is discussed in the next Section.

A reference distribution determined from a small-displacement-gradient analysis is adequate for most applications. However, accurate descriptions of fields near the zone of finite strains may be desirable in some applications, e.g. computational studies on the micromechanisms of ductile initiation. In such cases it is preferable to calculate $\left(\sigma_{\theta \theta}\right)_{\mathrm{SSY} ; \mathrm{T}=0}$ by a finite deformation analysis and to use (2.9) for the definition of $Q$. More importantly for practical applications, the evolution of stress triaxiality in a finite-width, cracked body can be evaluated for an actual stress-strain relation, not just the power-law relation, if the $\left(\sigma_{\theta \theta}\right)_{S S Y ; T=0}$ reference field for the MBL model is determined with the same stress-strain relation. This extends the applicability of the approach to a much broader range of material responses. In contrast, the reference field $\left(\sigma_{\theta \theta}\right)_{\mathrm{HRR}}$ is defined for an elastic power-law hardening material and the calculations in the finite body also must employ an elastic powerlaw hardening relation.

\subsection{Variation of $Q$ with Distance}

Because $Q$ scales the difference field relative to a reference stress state, it provides a sensitive measure of the evolution of near-tip stress triaxiality in finite width cracked bodies. It also can be used to detect changes in the stress triaxiality that deviates from the pattern that develops under MBL loadings. For this purpose, we consider $Q(F)$ defined by

$$
Q(F) \equiv \frac{\sigma_{\theta \theta}(\bar{F})-\left[\sigma_{\theta \theta}(\bar{F})\right]_{\mathrm{SSY} ; \mathrm{T}=0}}{\sigma_{0}}, \quad \text { at } \theta=0
$$

where $\bar{r} \equiv r /\left(J / \sigma_{0}\right)$. Note that $\left(\sigma_{\theta \theta}\right)_{\mathrm{SSY} ; \mathrm{T}=0}$ is chosen as the reference field.

The mean gradient of $Q$ over $1<F<5$,

$$
Q^{\prime}=\frac{Q(r=5)-Q(r=1)}{4},
$$

can be used to monitor changes in the pattern of the stress triaxiality ahead of the crack that do not conform to a spatially uniform hydrostatic stress field of adjustable magnitude. 
$Q^{\prime}$ provides a measure of the robustness of the $J-Q$ fields in the application of interest. For example, $Q^{\prime}=0.04$ means $\Delta \sigma_{\theta \theta}$ varies by less than $0.16 \sigma_{0}$ over the interval $1<\bar{r}<5$; that is, $\Delta \sigma_{\theta \theta}$ is effectively constant over those distances. On the other hand, an $\left|Q^{\prime}\right|$ much larger than 0.1 implies that the variation of $\Delta \sigma_{\theta \theta}$ over the interval $1<\bar{r}<5$ can be comparable to $\sigma_{0}$. This is unacceptably large if the theory is employed to predict cleavage fracture which is very sensitive to changes in the hoop stress.

Table 2.1: Reference stresses, $\sigma_{\theta \theta} / \sigma_{0}$, for MBL problem, $T / \sigma_{0}=0$.

\begin{tabular}{|c|c|c|c|c|}
\hline$n$ & $r /\left(J / \sigma_{0}\right)$ & HRR & Small Strain & Finite Strain \\
\hline \multirow{5}{*}{3} & 1 & 5.99 & 5.46 & 5.95 \\
\hline & 2 & 5.04 & 4.53 & 4.72 \\
\hline & 3 & 4.55 & 4.06 & 4.19 \\
\hline & 4 & 4.24 & 3.76 & 3.85 \\
\hline & 5 & 4.01 & 3.53 & 3.61 \\
\hline \multirow{5}{*}{5} & 1 & 4.77 & 4.42 & 4.83 \\
\hline & 2 & 4.25 & 3.90 & 4.06 \\
\hline & 3 & 3.97 & 3.63 & 3.73 \\
\hline & 4 & 3.79 & 3.44 & 3.52 \\
\hline & 5 & 3.65 & 3.29 & 3.36 \\
\hline \multirow{5}{*}{10} & 1 & 3.83 & $\overline{3.57}$ & 3.79 \\
\hline & 2 & 3.59 & 3.35 & 3.52 \\
\hline & 3 & 3.46 & 3.22 & 3.33 \\
\hline & 4 & 3.38 & 3.12 & 3.20 \\
\hline & 5 & 3.31 & 3.03 & 3.11 \\
\hline \multirow{5}{*}{$\infty$} & 1 & - & 2.83 & 2.50 \\
\hline & 2 & - & 2.80 & 2.97 \\
\hline & 3 & - & 2.77 & 2.91 \\
\hline & 4 & - & 2.74 & 2.86 \\
\hline & 5 & - & 2.71 & 2.82 \\
\hline
\end{tabular}

\subsection{Simplified Forms for Engineering Applications}

Two simplified representations for the Q-family of fields within the forward sector have been proposed by OS. The first is

$$
\sigma_{i j}=\left(\sigma_{i j}\right)_{\mathrm{HRR}}+Q \sigma_{0} \delta_{i j}
$$

where $\delta_{i j}$ is the Kronecker delta. This form is consistent with (2.7). The second form is

$$
\sigma_{i j}=\left(\sigma_{i j}\right)_{\mathrm{SSY} ; \mathrm{T}=0}+Q \sigma_{0} \delta_{i j}
$$

which is consistent with (2.9). The physical interpretation of (2.13) and (2.14) is this: negative (positive) $Q$ values mean that the hydrostatic stress ahead of the crack is reduced (increased) by $Q \sigma_{0}$ from the $J$-dominant stress state, or the standard small scale yielding stress state. This interpretation is precise when $\left|Q^{\prime}\right| \ll 1$.

A operational definition of $Q$ consistent with its interpretation as a triaxiality parameter is

$$
Q_{m} \equiv \frac{\sigma_{m}-\left(\sigma_{m}\right)_{\mathrm{SSY} ; \mathrm{T}=0}}{\sigma_{0}} \quad \text { at } \theta=0, \quad r=2 J / \sigma_{0}
$$


where $\sigma_{m}$ is the hydrostatic stress. We have calculated $Q$ based on the hoop stress (2.9) and the mean stress (2.15) for the full range of $T$-stresses and finite width geometries. The difference between $Q$ and $Q_{m}$ is always less than 0.1 .

Our numerical studies show that $(2.14,2.15)$ provide a more accurate representation of the full range of near-tip fields so that a fracture methodology based on $(2.14,2.15)$ has a greater range of validity. $Q$ values presented in this paper are based on the definition in (2.9). To simplify subsequent discussions, the term small-scale yielding (SSY) will refer to

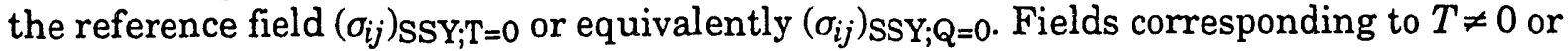
$Q \neq 0$ will be explicitly stated as such.

\subsection{Difference Field and Higher-Order Terms of the Asymptotic Series}

The connection between the difference field and higher-order terms of the asymptotic series can be understood in the context of the MBL formulation. Here the stress fields obey the functional form

$$
\sigma_{i j}=\sigma_{0} f_{i j}\left(\frac{r}{J / \sigma_{0}}, \theta ; Q\right)
$$

which also should apply to finite-width crack geometries as long as the characteristic crack dimension $L$ is sufficiently large compared to $J / \sigma_{0}$. Now, if one assumes a product dependence on the first argument in (2.16) and works with deformation plasticity theory with power law hardening behavior, then one obtains a series in $r /\left(J / \sigma_{0}\right)$ :

$$
\sigma_{i j}=\sigma_{0}\left(\frac{J}{\alpha \varepsilon_{0} \sigma_{0} I_{n} r}\right)^{1 /(n+1)} \tilde{\sigma}_{i j}(\theta, n)+\text { second-order terms + higher-order terms }
$$

where $\varepsilon_{0}$ is a reference strain, $\alpha$ a material constant (equal to unity for the stress-strain response defined by (2.10)) and $I_{n}$ is an integration constant. By definition, the asymptotic series beyond the first term is equivalent to the difference field since (see Section 2.2 )

$$
\sigma_{i j}=\left(\sigma_{i j}\right)_{\mathrm{HRR}}+\text { difference field . }
$$

Thus the HRR field and (only) the second-order term provide a two-term approximation to the solution for the MBL problem and this point appears not always to be understood.

The higher order asymptotic analysis of Li and Wang [17] and Sharma and Aravas [18] has been extended by Xia, Wang and Shih [19]. They have obtained a five term expansion for the series in (2.17) for $n=3$ and a four term series for $n=10$. The four term series accurately matches the radial and angular variations of the difference field given in Fig 3 and Fig. 5 by O'Dowd and Shih [13] for an $n=10$ material. Indeed, in the forward sec$\operatorname{tor}|\theta|<\pi / 2$, the collective behavior of the second, third and fourth order terms is effectively equivalent to a spatially uniform hydrostatic stress state so that (2.17) can be approximated by the simpler form in (2.13).

Finally, it may be noted that an admissible range of stress states for an elastic-perfectly plastic material can be written in the form

$$
\sigma_{i j}=\left(\sigma_{i j}\right)_{\text {Prandtl }}+Q \sigma_{0} \delta_{i j},|\theta| \leq \pi / 4,
$$

where $\left(\sigma_{i j}\right)_{\text {Prandlt }}$ designates the Prandtl slip-line solution and the difference field corresponds simply to a uniforn hydrostatic stress state scaled by $Q[14,25]$. 


\section{$2.7 J-Q$ Material Toughness Locus}

The $J-Q$ theory provides the quantitative framework to characterize a material's fracture resistance over a range of crack-tip stress triaxiality. The experimental determination of the toughness iocus and its utilization in engineering applirations are discussed below.

The competition between fracture by cleavage and ductile tearing controls the fracture resistance of ferritic steels in the ductile-to-brittle transition region. Now consider test conditions where both mechanisms are operative. Fracture by (stress-controlled) cleavage generally requires higher crack-tip constraint while ductile tearing develops at low constraint; this is illustrated by the two distinct segments to the toughness locii shown in Fig. 2.3a. Since measured toughness values generally exhibit scatter, both the lower and higher toughness locii are indicated which define bands for brittle and ductile failure. Toughness values over the full range of crack-tip constraints can be measured by using the test geometries depicted in Fig. 2.3a. As an example, deeply-cracked SE(B) specimens generate high crack-tip stress triaxiality, i.e., $Q \approx 0$. They produce driving force curves which rise steeply and therefore intersect the toughness locii within a well-defined, narrow zone of the $J-Q$ diagram. In contrast, center-cracked panels and single-edge cracked panels loaded in terision are low constraint crack geometries. They produce driving force curves which rise with more shallow slopes and thus intersect the toughness locii over a broad zone in the $J-Q$ diagram. The shallow driving force curves of low constraint geometries imply considerably greater scatter in cleavage toughness values $\left(J_{c}\right)$, a phenomenon commonly observed in testing $\mathrm{SE}(\mathrm{B})$ specimens with small $a / W$ ratios, for example.

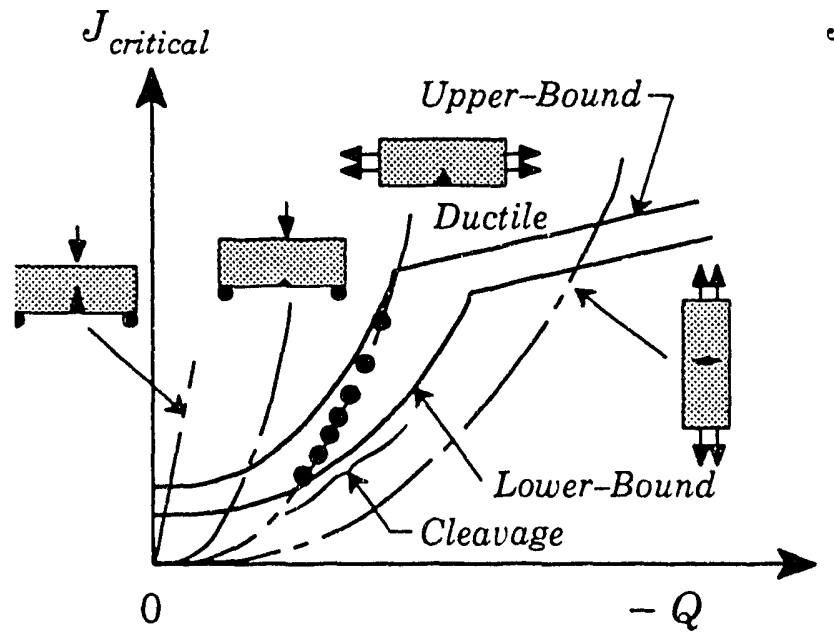

(a) Laboratory Testing

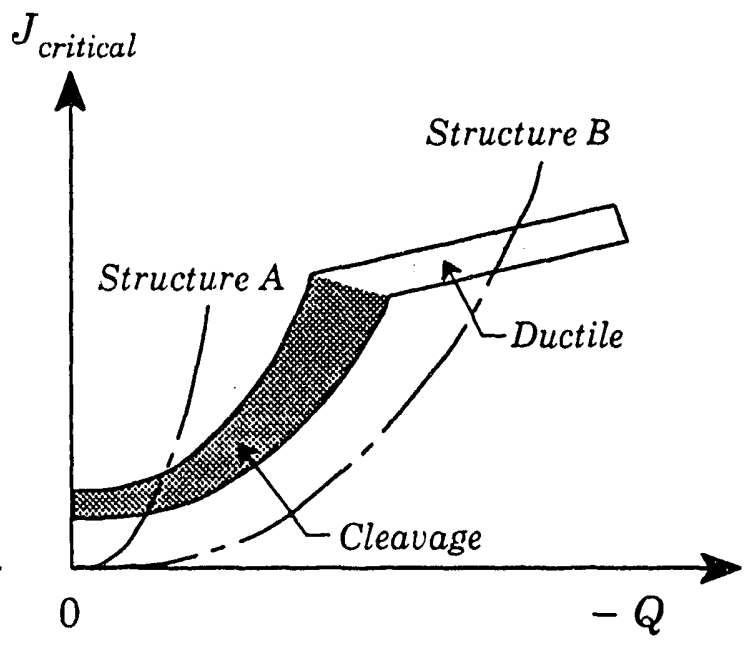

(b) Fracture Assessment

Figure 2.3 Application of the $J-Q$ methodology in fracture assessments. (a) Laboratory testing of specimens with varying constraint to measure the material's fracture resistance. Circles indicate anticipated scatter which define upper-lower bounds. (b) Evaluation of structural flaws using measured toughness locus and predicted $J-Q$ response for two structural configurations. Cleavage fracture is predicted for Structure A; ductile tearing is predicted for Structure B.

Utilization of the toughness locus in fracture assessments is illustrated in Fig. 2.3b. Suppose that the material's fracture resistance under service conditions is characterized by the indicated cleavage-ductile failure band. The driving force curve for a structure of 
high crack-tip constraint, structure $A$, rises rapidly in the $J-Q$ space so that cleavage fracture occurs when the driving force curve intersects the failure locus. In contrast a low constraint geometry such as structure $B$, induces a gradually rising driving force curve so that ductile tearing is the likely event at overload. 


\section{MICROMECHANICAL CONSTRAINT CORRECTIONS}

Dodds and Anderson [31,32] show that by quantifying the effects of finite size on the relationship between microscale crack driving force (e.g. near-tip stresses and strains) and macro-scale crack driving force (e.g. $J$, CTOD), the apparent size effect on fracture toughness can be predicted rigorously without resort to empirical arguments. These size effects become steadily more pronounced as load increases due to the deviation of crack-tip region deformations from the small scale yielding conditions essential for single parameter fracture mechanics (SPFM) to apply. When SPFM becomes invalid, a micromechanics failure criteria is required to establish the near-tip conditions at fracture. Finite element analysis, or alternatively the near-tip stresses described by the $J-Q$ theory, provides a means to quantify the geometry dependent relations between these micromechanical failure conditions and macro-scale crack driving force. This permits (in principle) prediction of fracture in any body from toughness values measured using standard specimens.

For steels operating at temperatures where cleavage occurs after significant plastic deformation but before the initiation of ductile growth (lower to mid-transition), attainment of a critical stress over a microstructurally relevant volume is an appropriate micromechanical failure criteria [21-39]. A number of important engineering structures can fail by this mechanism, including high strength rails, offshore oil platforms, ships, storage tanks, and nuclear pressure vessels after years of neutron irradiation embrittlement. Techniques for predicting the apparent size effects on cleavage fracture toughness developed by Dodds and Anderson are described in the following sections.

\subsection{Transgranular Cleavage Mechanism}

A number of micromechanical models for transgranular cleavage fracture have been proposed, most derive from weakest-link statistics. The weakest-link models assume the largest or most favorably oriented fracture-triggering particle controls the cleavage failure. The actual trigger event involves a local Griffith instability of a microcrack which forms at a microstructural feature such as a carbide or inclusion; satisfaction of of the Griffith energy balance occurs when the critical stress is reached in the vicinity of the microcrack. The size and location of the triggering microstructural feature(s) dictate the fracture toughness and produces the scatter routinely observed in results of cleavage fracture tesis.

The Griffith instability criterion implies fracture at a critical normal stress near the crack tip; the statistical sampling aspect of the mechanism (i.e., the probability of finding a triggering microfeature near the crack tip) suggests a dominant role for the volume of material within a process-zone over which the opening mode stress exceeds a threshold value sufficient to initiate cleavage. The probability of cleavage fracture in a cracked specimen may then be expressed in the following general form:

$$
F=F\left[V\left(\sigma_{1}\right)\right]
$$

where $F$ is the failure probability, $\sigma_{1}$ is the maximum principal stress at a point and $V\left(\sigma_{1}\right)$ is the cumulative volume sampled over which the principal stress is equal to or greater than $\sigma_{1}$. This form of $F$ applies to any fracture process controlled by maximum principal stress, not just weakest-link failure which is now being questioned $[40,41]$. In particular, the $F$ criterion of (3.1) does not require material-specific assumptions for the distribution and strength of cleavage triggering particles.

Unlike other micromechanics models, the present methodology does not attempt to predict absolute values of $J_{c}$ from metallurgical parameters that describe the distribution and 
3 trength of cleavage triggering particles. Rather, the micromechanical model predicts the variation of fracture toughness with constraint changes for a given material/temperature by scaling to a reference condition. The crack-tip stress fields in a test specimen are compared to the limiting solution of SSY. A $J$-like parameter, denoted $J_{0}$, is obtained from this comparison to the reference solution. $J_{0}$ is the $J$ to which the SSY model (infinite body) must be loaded to achieve the same stressed volume, and thereby the same likelihood of cleavage fracture, as in a finite body.

A critical value of $J_{0}$ represents the fracture toughness of an infinitely large specimen; the ratio of applied $J / J_{0}>1$ implies that the specimen has experienced a constraint loss that causes the commonly observed increase in measured fractured toughness.

\subsection{Development of Constraint Corrections}

By employing the family of near-tip states in the form of (2.4), the maximum principal stress also has the form

$$
\frac{\sigma_{1}}{\sigma_{0}}=f_{1}\left(\frac{r}{J_{0} / \sigma_{0}}, \theta ; Q\right) \text {. }
$$

For any given value of $Q$ and $\theta, \sigma_{1} / \sigma_{0}$ decreases monotonically once $r$ extends beyond the finitely deformed region of $r \leq J / \sigma_{0}$. Rearrangement of the above expression furnishes a relation for the distance $r$ as a function of $\theta$ and $\sigma_{1} / \sigma_{0}$ as

$$
r=\frac{J}{\sigma_{0}} g_{1}\left(\theta ; \sigma_{1} / \sigma_{0}, Q\right) \text {. }
$$

Consider a particular level of the principal stress $\sigma_{1} / \sigma_{0}$. The area $A$ over which the principal stress is greater than $\sigma_{1} / \sigma_{0}$ is given by

$$
A=\frac{J^{2}}{\sigma_{0}^{2}} h\left(\sigma_{1} / \sigma_{0} ; Q\right), \quad h=\frac{1}{2} \int_{-\pi}^{\pi} g_{1}^{2}\left(\theta ; \sigma_{1} / \sigma_{0}, Q\right) d \theta .
$$

The area enclosed by the contour of level $\sigma_{1} / \sigma_{0}$ depends on $J$ as well as the triaxiality of the near-tip fields identified with $Q$. To fix ideas, let $A_{0}$ and $J_{0}$ designate the area and $J$ associated with the $Q=0$ field, and let $A_{\mathrm{FB}}$ and $J_{\mathrm{FB}}$ designate the area and $J$ associated with a crack in a finite body with $Q \neq 0$. Then we have

$$
A_{0}=\frac{J_{0}^{2}}{\sigma_{0}^{2}} h_{0}\left(\sigma_{1} / \sigma_{0}\right) ; \quad h_{0}=\frac{1}{2} \int_{-\pi}^{\pi} g_{1}^{2}\left(\theta ; \sigma_{1} / \sigma_{0}, Q=0\right) d \theta
$$

and

$$
A_{\mathrm{FB}}=\frac{J_{\mathrm{FB}}^{2}}{\sigma_{0}^{2}} h_{\mathrm{FB}}\left(\sigma_{1} / \sigma_{0}\right) ; \quad h_{\mathrm{FB}}=\frac{1}{2} \int_{-\pi}^{\pi} g_{1}^{2}\left(\nu ; \sigma_{1} / \sigma_{0}, Q\right) d \theta .
$$

Upon initial loading of the finite body, $Q=0$ so that $h_{\mathrm{FB}}=h_{0}$; compare expressions (3.5b) and (3.6b). As the load increases, plasticity spreads over the body, $Q$ becomes non-zero, and $h \mathrm{FB}$ begins to deviate from $h_{0}$.

For a given material and temperature, the present micromechanics model requires the attainment of equivalent stressed volumes $\left(A_{\mathrm{FB}} \times\right.$ thickness $B$ ) for cleavage fracture in different specimens. The ratio of applied $J$-values in a finite body and the reference $Q=0$ stress state that generate equivalent stressed volumes is found by equating areas in (3.5) and (3.6) to yield 


$$
\frac{J_{\mathrm{FB}}}{J_{0}}=\sqrt{\frac{h_{0}\left(\sigma_{1} / \sigma_{0}\right)}{h_{\mathrm{FB}}\left(\sigma_{1} / \sigma_{0}\right)}} .
$$

The results thus far are generally applicable and do not rely upon any particular form of the $J-Q$ fields.

The $J$ ratios are evaluated using (3.7) at each loading level and for a range of principal stress values. The ratio quantifies the size and geometry dependence of cleavage fracture toughness. Consider, for example, a test specimen that fails at $J_{\mathrm{c}}=200 \mathrm{kPa} \cdot \mathrm{m}$. Suppose the computed ratio $J_{\mathrm{FB}} / J_{0}=2$ at fracture $\left(J_{\mathrm{FB}}=\mathrm{e}_{\mathrm{c}}\right)$ in the test specimen; then a very large specimen made from the same material and tested at the same temperature is predicted to fail at $J_{c}=100 \mathrm{kPa} \cdot \mathrm{m}$. Similarly, the fracture toughness ratios for test specimens with the same absolute size but varying crack-deptiss to specimen-widths, $a / W$, may be quantified. The model predicts a sharp increase in fracture toughness with decreasing $a / W$ ratio.

\section{Self-Similar Principal Stress Contours}

The character of the near-tip fields has been investigated by O'Dowd and Shih [13,14] and Xia, Wang and Shih [19]. From their results, e.g., (2.14), we write

$$
\begin{aligned}
\frac{\sigma_{1}}{\sigma_{0}} & =f_{1}\left(\frac{r}{J_{0} / \sigma_{0}}, \theta ; Q\right) \\
& =f_{0}\left(\frac{r}{J_{0} / \sigma_{0}}, \theta\right)+Q
\end{aligned}
$$

where the form in (3.8b) describes the fields in the forward sector, $|\theta|<\pi / 2$ and $r<5 \mathrm{~J} / \sigma_{0}$. Detailed computational studies have shown that principal stresses of sufficiently high level, say $\sigma_{1} / \sigma_{0}>2.0$, are found only in ise forward sector. The form in $(3.8 \mathrm{~b})$ is applicable in the preceding micromechanics analysis if we confine attention to $\sigma_{1} / \sigma_{0}>2.0$. Rearranging $(3.8 \mathrm{~b})$ yields

$$
\frac{\sigma_{1}}{\sigma_{0}}-Q=f_{0}\left(\frac{r}{J_{0} / \sigma_{0}}, \theta\right) .
$$

The form in (3.9) constitutes a self-similar field for $\sigma_{1} / \sigma_{0}-Q$. Moreover, the behavior of $\sigma_{1} / \sigma_{0}-Q$ obeys the form governing the $Q=0$ reference solution, which has been determined by small-scale yielding analysis.

To understand the implications of (3.9), we focus on a particular value of $\sigma_{1} / \sigma_{0}$, say $\sigma_{1} / \sigma_{0}=3$. Consider the behavior of $\sigma_{1} / \sigma_{0}-Q$ as the deformation level, measured by $J$, increases. Suppose for the moment that $Q$ remains constant; then the contour for a fixed level of $\sigma_{1} / \sigma_{0}-Q$, presented in the normalized distances $X /\left(J / \sigma_{0}\right)$ and $Y /\left(J / \sigma_{0}\right)$, remains unaltered in size and shape with increasing deformation level. As an example, the outermost contour in Fig. 3.1 corresponding to the reference solution, fixed $Q \equiv 0$, maintains its size and shape as the deformation level is increased.

Now let $Q$ evolve with increasing deformation as happens in a finite size body. $Q$ decreases gradually corresponding to a loss of stress triaxiality as the deformation level increases; $\sigma_{1} / \sigma_{0}-Q$ must increase since $\sigma_{1} / \sigma_{0}$ is fixed. Therefore the evolution (size reduction) of a contour, associated with a fixed value of $\sigma_{1} / \sigma_{0}$, under increasing plastic yielding can be described by contours, associated with increasingly higher levels of $\sigma_{1} / \sigma_{0}-Q$, governed by (3.9). The sequence of diminishing contours associated with increasing levels of $\sigma_{1} / \sigma_{0}-Q$, corresponding to a fixed level of $\sigma_{1} / \sigma_{0}=3$, is depicted in Fig. 3.1 for a shallow notch $\mathrm{SE}(\mathrm{B})$ specimen. 


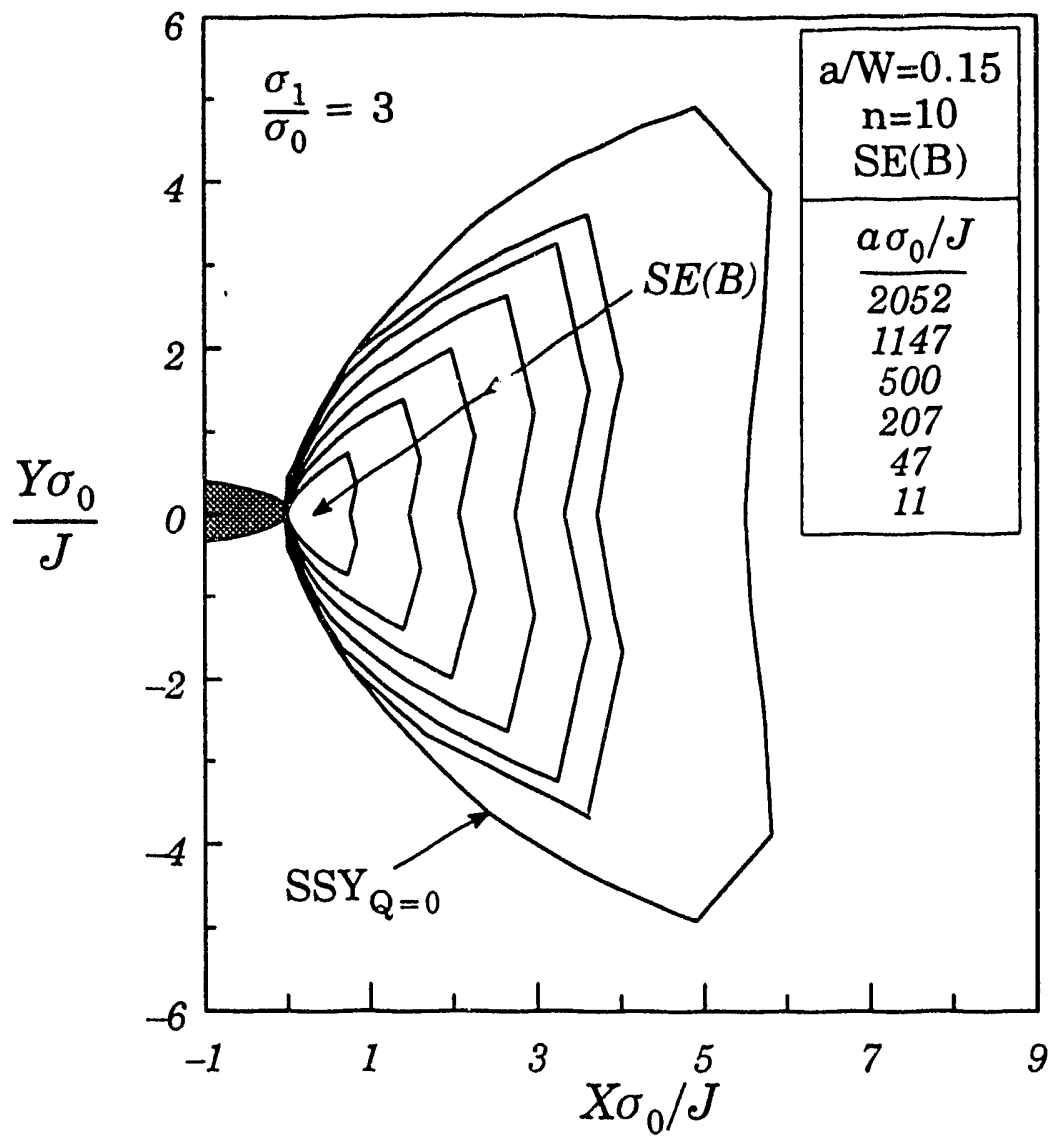

Figure 3.1 Comparison of a maximum principal stress contour for $\mathrm{SSY}_{Q=0}$ with those for an $a / W=0.15, n=10 \mathrm{SE}(\mathrm{B})$. $\mathrm{SE}(\mathrm{B})$ contours decrease in size with increasing deformation (i.e. with decreasing $a \sigma_{0} / J$ ).

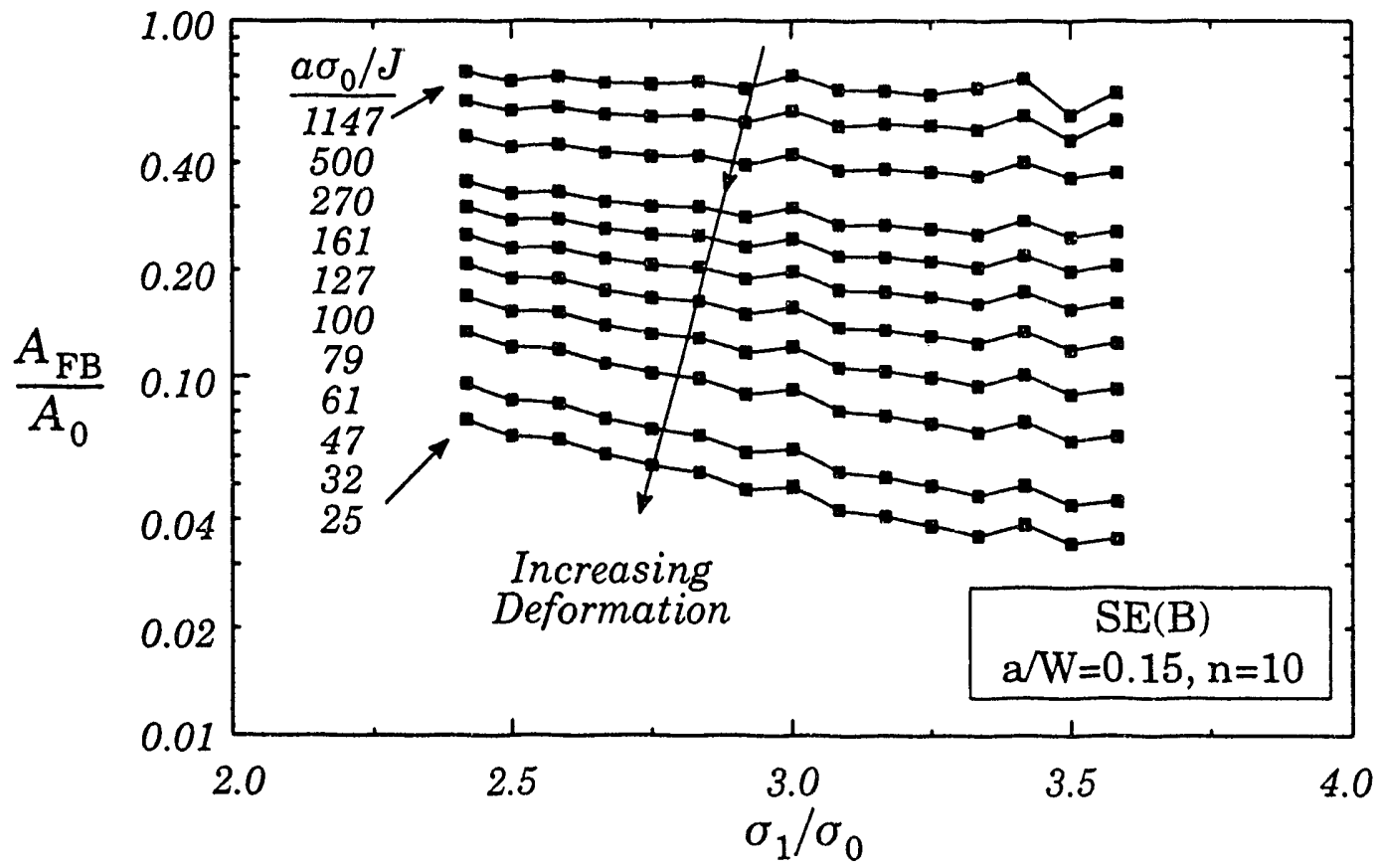

Figure 3.2 Areas within principal stress contours for an $a / W=0.15, n=10 \mathrm{SE}(\mathrm{B})$. Values are normalized by area within contour for $\mathrm{SSY}_{Q=0}$ at same $J$-value. 
Finally, the asymptotic studies show $f_{0}$ is nearly separable in $r$ and $\theta$ when $r /\left(J / \sigma_{0}\right)$ is sufficiently small (HRR field). Consequently, the shape of a contour is maintained as its size diminishes. The self-similarity of $\sigma_{1} / \sigma_{0}-Q$ prevails only to the extent that the $J-Q$ form in (2.14) remains applicable.

\section{Inverted Relationships}

A specialized form of (3.3) is developed by noting the dependence of $r$ on $\sigma_{1} / \sigma_{0}$ and $Q$ involve them in the combination $\sigma_{1} / \sigma_{0}-Q$, i.e.,

$$
r=\frac{J}{\sigma_{0}} g_{1}\left(\theta ; \sigma_{1} / \sigma_{0}-Q\right) \text {. }
$$

The results in (3.4) through (3.7) are simplified by using the form in (3.10).

The key question to resolve with this approach concerns the sensitivity of the $A_{\mathrm{FB}} / A_{0}$ and $J_{\mathrm{FB}} / J_{0}$ ratios to $\sigma_{1} / \sigma_{0}$. Let $J_{\mathrm{FB}}^{*}$ denote the value associated with $\left(\sigma_{1} / \sigma_{0}\right)^{*}$. Then to firstorder,

$$
\frac{J_{\mathrm{FB}}-J_{\mathrm{FB}}^{*}}{J_{0}} \propto Q\left[\left(\frac{\sigma_{0}}{\sigma_{1}}\right)^{*}-\left(\frac{\sigma_{0}}{\sigma_{1}}\right)\right] .
$$

When $Q \approx 0$, the ratio $J_{\mathrm{FB}} / J_{0}$ is insensitive to $\sigma_{1} / \sigma_{0}$ since the quantity in [] is scaled by $Q$. When $Q$ is large (negative), the $J_{\mathrm{FB}} / J_{0}$ exhibits a small sensitivity to $\sigma_{1} / \sigma_{0}$. The weak dependence of $J_{\mathrm{FB}} / J_{0}$ on $\sigma_{1} / \sigma_{0}$ has been confirmed by analyzing the evolution of near-tip fields in common fracture specimens. Figures 3.1-3.3 provide typical results obtained through finite element modeling. The specimen is a single-edge notched bend bar containing a shallow notch, $a / W=0.15$, with a strain hardening exponent of $n=10$. Figure 3.2 shows the area enclosed by principal stress contours $\left(A_{\mathrm{FB}}\right)$. The $\mathrm{SE}(\mathrm{B})$ areas are normalized by the area, $A_{0}$, defined by the same contour of the reference solution $(Q=0)$ when loaded to the same $J$ as the $\mathrm{SE}(\mathrm{B}) ; J_{0}=J_{\mathrm{FB}}$. The area ratios remain relatively insensitive to $\sigma_{1} / \sigma_{0}$ until the deformations become excessive. $J_{0}$ is calculated for each line of this figure using (3.7). $J_{\mathrm{FB}} / J_{0}$ ratios are independent of the principal stress selected for computation over a wide range as shown in Fig. 3.3. In practice, the computation of $J_{\mathrm{FB}} / J_{0}$ ratios is terminated when the values differ by more than $10 \%$ at the smallest and largest principal stress values as indicated on the figure. A larger deviation indicates breakdown in the similarity of the SE(B) and SSY stress fields and thus an unacceptably large dependence on the critical fracture stress.

To simplify applications of this methodology, the SSY areas within principal stress contours are expressed as a function of principal stress $\left(\sigma_{1}\right)$ and strain hardening coefficient $(n)$ as:

$$
\frac{A_{0} \sigma_{0}^{2} \varepsilon_{0}^{2} \alpha^{2}}{J_{0}^{2}}=10^{\beta} / 250000
$$

where the curve fitting function is given by

$$
\beta\left(\frac{\sigma_{1}}{\sigma_{0}}, n\right)=H_{0}+H_{1}\left(\frac{\sigma_{1}}{\sigma_{0}}\right)+H_{2}\left(\frac{\sigma_{1}}{\sigma_{0}}\right)^{2}+H_{3}\left(\frac{\sigma_{1}}{\sigma_{0}}\right)^{3}+H_{4}\left(\frac{\sigma_{1}}{\sigma_{0}}\right)^{4}
$$

with fittir.g coefficients $H_{i}$ given in Table 3.1 for a range of hardening exponents. 


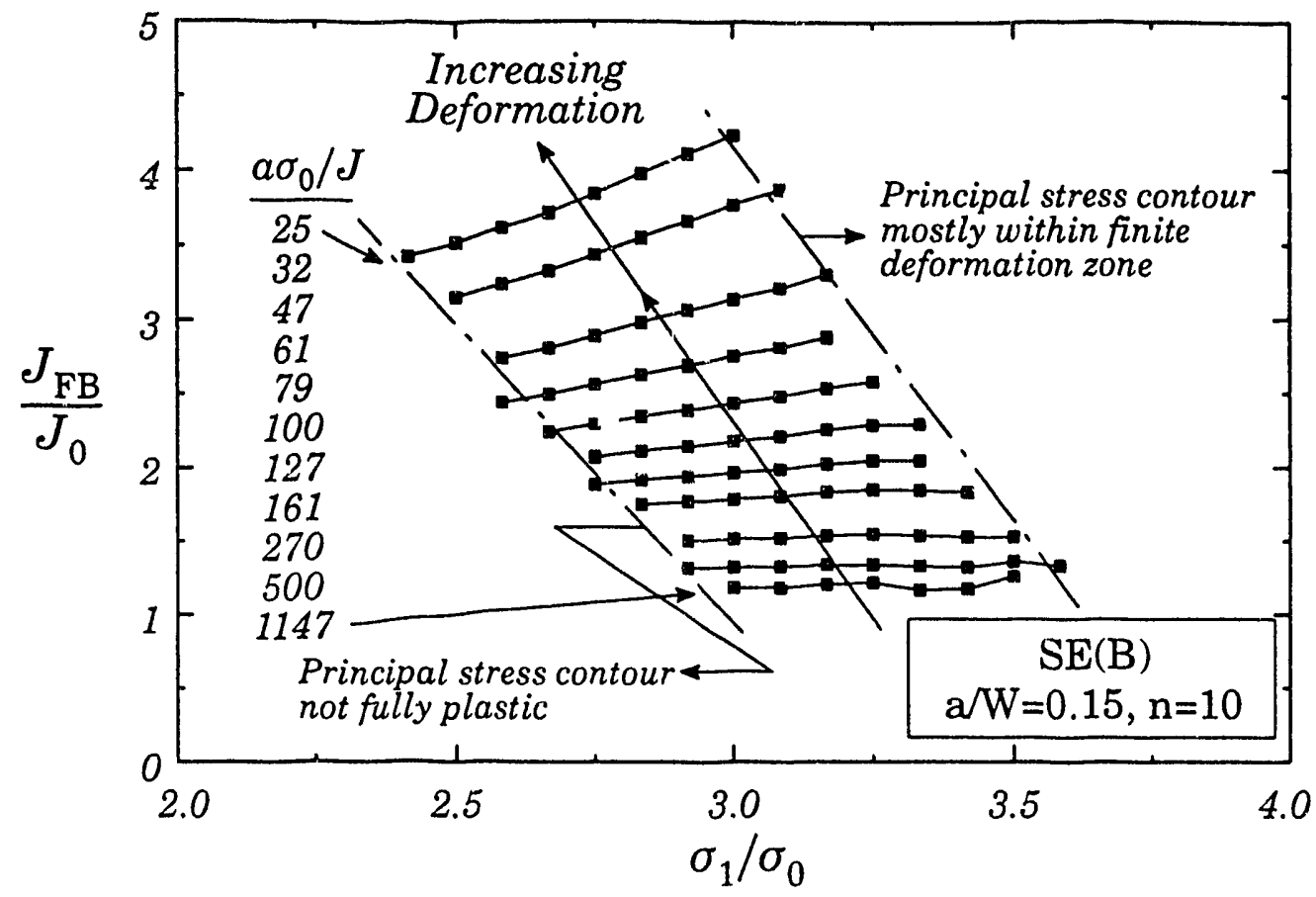

Figure 3.3 Influence of specified critical stress on the micromechanics prediction of fracture toughness variation with constraint for an $a / W=0.15, n=10 \mathrm{SE}(\mathrm{B})$.

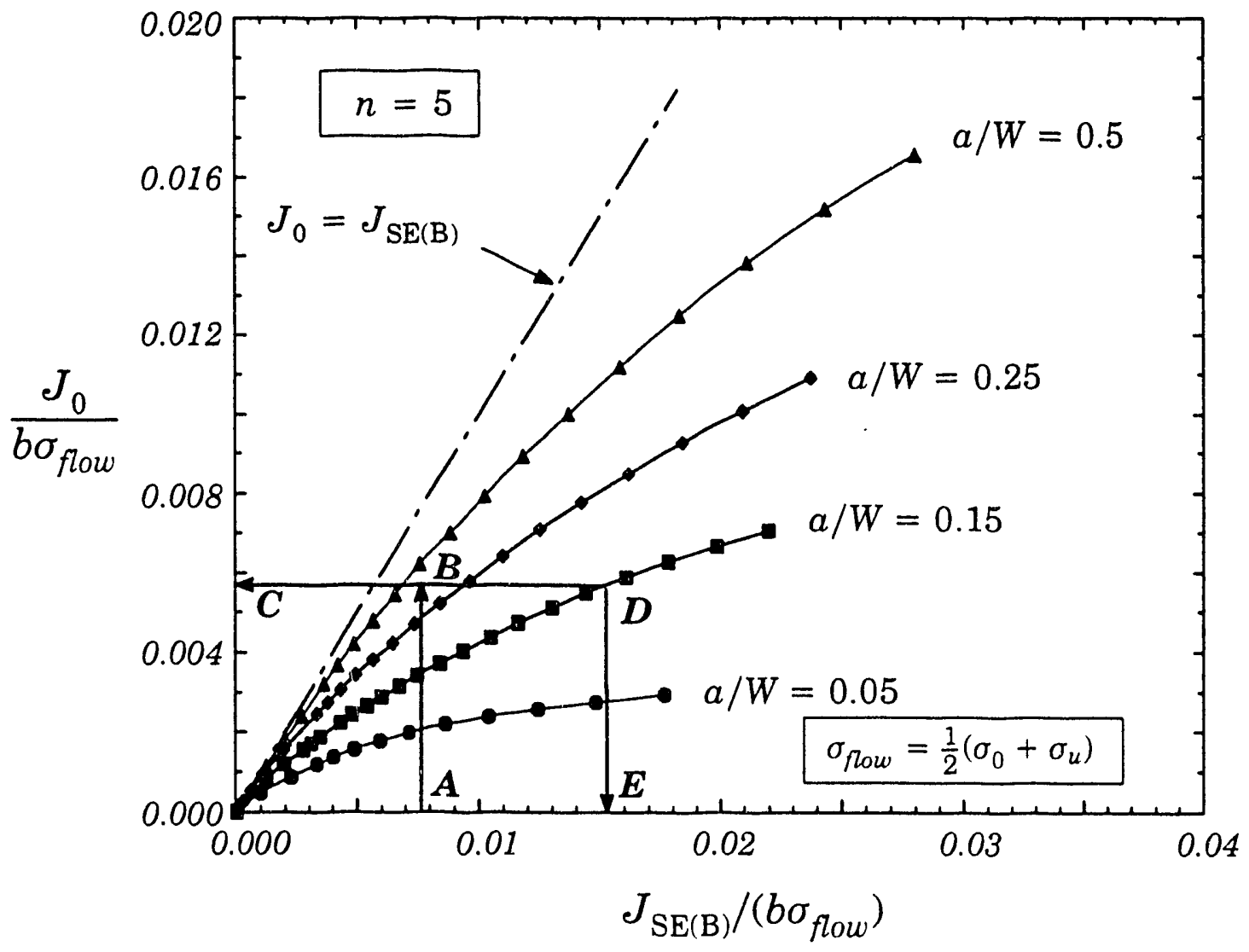

Figure 3.4 Micromechanics prediction of $a / W$ effects on cleavage fracture toughness for an $n=5, \mathrm{SE}(\mathrm{B})$ fracture specimen. 


\begin{tabular}{|c|c|c|c|c|c|c|c|}
\hline \multicolumn{7}{|c|}{ Table 3.1: Fit coefficients for (3.13) } \\
\hline$n$ & $H_{0}$ & $H_{1}$ & $H_{2}$ & $H_{3}$ & $H_{4}$ & $\begin{array}{c}\text { Mini- } \\
\text { mum } \\
\sigma_{1} / \sigma_{0}\end{array}$ & $\begin{array}{c}\text { Maxi- } \\
\text { mum } \\
\sigma_{1} / \sigma_{0}\end{array}$ \\
\hline 4.0 & 6.4306 & -2.4711 & 0.5037 & -0.07975 & 0.00552 & 2.0 & 4.0 \\
\hline 5.0 & 6.2579 & -2.1653 & 0.3749 & -0.06603 & 0.00505 & 2.0 & 4.0 \\
\hline 10.0 & 7.6641 & -4.3138 & 1.7368 & -0.43685 & 0.03560 & 2.0 & 3.6 \\
\hline 20.0 & -3.2613 & 14.4338 & -10.2659 & 3.01033 & -0.34420 & 2.0 & 3.2 \\
\hline
\end{tabular}

These expressions are obtained by curve fitting the results of small-displacement gradient, finite element analyses conducted on the MBL problem with $T / \sigma_{0}=0$. The material stressstrain curve employed in the analyses follows the conventional Ramberg-Osgood model given by

$$
\frac{\varepsilon}{\varepsilon_{0}}=\frac{\sigma}{\sigma_{0}}+\alpha\left(\frac{\sigma}{\sigma_{0}}\right)^{n}
$$

which exhibits a slightly different behavior than the elastic power-law model defined in (2.10).

\subsection{Application of the Constraint Corrections in Fracture Testing}

The computational procedures outlined above have been applied to generate $J_{\mathrm{FB}} / J_{0}$ ratios for a variety of test specimens and material properties [30,31]. Figure 3.4 provides the results of such computations for $S E(B)$ specimens having a range of $a / W$ ratios modelled with an $n=5$ strain hardening material. Values of $J_{\mathrm{FB}}$ and $J_{0}$ are plotted on separate axes to facilitate removal of the size effect in experimental data. Points on the curves describe $\left(J_{\mathrm{FB}}, J_{0}\right)$ pairs that produce equal stressed volumes of material in the finite--size test specimen and in the SSY model. Upon initial loading, crack-tip plasticity is well contained within a surrounding elastic field and identical values for $J_{\mathrm{FB}}$ and $J_{0}$ correspond to the same stressed volume of material at the crack tip. This $1: 1$ line is shown on the figure for reference. At higher loads and as constraint relaxes under extensive plastic flow, the finite-size test specimen requires more applied- $J\left(J_{\mathrm{FB}}>J_{0}\right)$ to achieve the same conditions for cleavage (same stressed volume) as in SSY.

Information of this type is useful for both analysis of fracture test data and for assessing the defect integrity of structures. Path A-B-C on Fig. 3.4 illustrates the procedure to remove geometry dependence from experimental cleavage fracture toughness data $\left(J_{c}\right.$ value at A) by determining the geometry independent cleavage fracture toughness $\left(J_{0}\right.$ value at C) corresponding to a measured $J_{c}$ value. Alternatively, Fig. 3.4 permits determination of the apparent fracture toughness for an $\mathrm{SE}(\mathrm{B})$ with any $a / W$ ratio from a known, $J_{0}$ value (path C-D-E for example).

Figure 3.5a shows the $J_{c}$ values measured by Sumpter and Forbes [10] for a BS4360 $43 \mathrm{~A}$ steel $(n \approx 5)$ using $\mathrm{SE}(\mathrm{B})$ specimens tested over a wide range of $a / W$ ratios. The data readily demonstrate the dramatic increase in cleavage fracture toughness with decreasing $a / W$ ratio. To remove the constraint effect on toughness, each experimental data point is processed using a path similar to A-B-C in Fig. 3.4 to obtain the corresponding $J_{0}$ value. Figure $3.5 \mathrm{~b}$ shows these "constraint corrected" toughness values. The toughness variation with $a / W$ ratio is effectively removed with this technique. The small remaining scatter in 
the experimental data for different $a / W$ ratios may be attributed to true metallurgical variations in the material and the unavoidable procedural variations in testing a large number of specimens. Application of this technique to other materials including A36, A515 and A533B [42] have been equally successful in removing the geometry dependence of $J_{c}$ values.

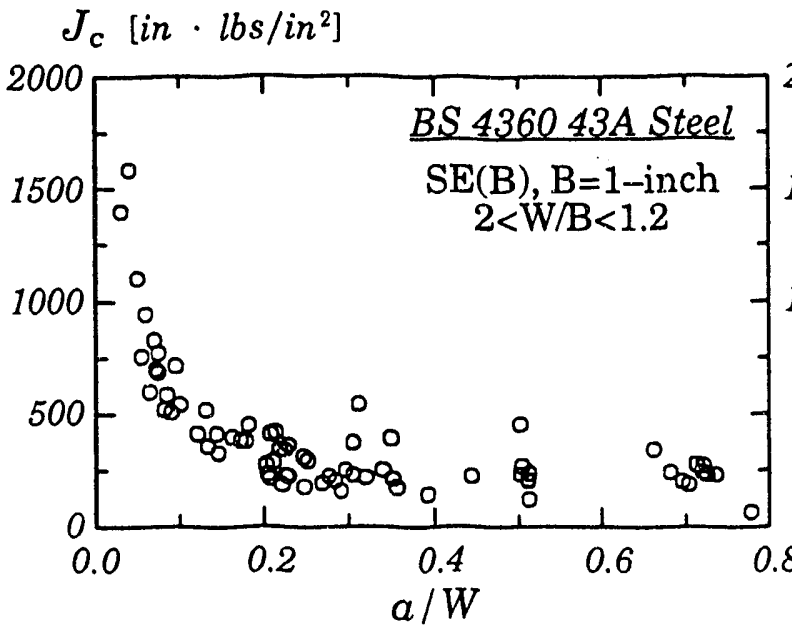

(a)

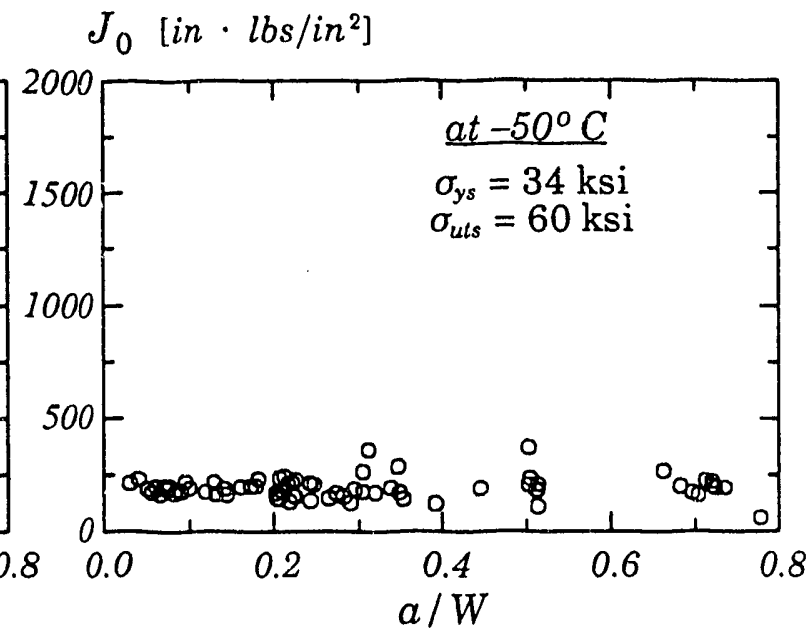

(b)

Figure 3.5 (a) Effect of initial crack depth on cleavage fracture toughness in a mild steel (Sumpter and Forbes [10]); (b) $J_{0}$ values (specimen size independent fracture toughness) calculated from experimental $J_{c}$ data using the micromechanics constraint correction.

\subsection{Engineering Use of $J-Q$ Fields in the Micromechanics Model}

When available, a $J-Q$ description of the crack-tip stresses for a test specimen or structural component may be readily employed to generate the constraint corrections for fracture toughness of the type shown in Fig. 3.4. Here we outline a procedure that is computationally simpler than the stressed volume approach defined by (3.2)-(3.7) but which yields essentially the same result.

Figure 3.6 shows the variation of opening mode stress on the crack plane with distance from the crack tip for several deformation levels for an $S E(B)$ specimen with $a / W=0.15$, $n=10$. The SE(B) stresses are normalized by the stress in the SSY model at the same relative distance ahead of the crack tip when the SSY model is loaded to the same $J$ as the $\mathrm{SE}(\mathrm{B})$. Distances are normalized by the similarity length-scale $r /\left(J /\left(\alpha \sigma_{0} \varepsilon_{0}\right)\right)$. The independence of these normalized stresses with distance from the crack tip indicates again the similarity of the SSY and SE(B) stress distributions. $J_{0}$ is calculated at a number of points along each line on this graph as the $J$ value required in the SSY model to achieve the same opening mode stress as in the finite body. The following equation is solved iteratively for $J_{0}$ using a simple nonlinear root solver:

$$
\frac{\left(\sigma_{\theta \theta}\right)_{\mathrm{FB}}}{\sigma_{0}}=\frac{\left(\sigma_{\theta \theta}\right)_{\mathrm{SSY}}}{\sigma_{0}} \text {, at } \theta=0
$$

where a closed-form fit to the crack-plane stresses in SSY is given by

$$
\frac{\left(\sigma_{\theta \theta}\right)_{\mathrm{SSY}}}{\sigma_{0}}=G_{1}(\hat{r})^{G_{2}} \exp \left(G_{3} \hat{r}\right)
$$

where 


$$
\begin{array}{ll}
\left(\sigma_{\theta \theta}\right)_{\mathrm{FB}} & \text { stress at distance } r \text { from the tip at loading } J_{\mathrm{FB}} \text { in finite body; } \\
\hat{r} & \text { normalized distance from tip: } \hat{r}=\frac{r}{J_{0} / a \sigma_{0} \varepsilon_{0}} \\
C_{\mathrm{i}} & \text { SSY fit coefficients summarized in Table 3.2. }
\end{array}
$$

The finite-body stresses needed in (3.15) are given by

$$
\frac{\left(\sigma_{\theta \theta}\right)_{F B}}{\sigma_{0}}=\frac{\left(\sigma_{\theta \theta}\right)_{\text {SSY }}}{\sigma_{0}}+Q(r)
$$

where the SSY term in (3.17) is given by the expression on the right side of (3.16) with $J_{0}$ replaced by $J_{\mathrm{FB}}$. The potential for a small radial dependence of $Q$ under large-scale yielding is included in (3.17). The $J_{\mathrm{FB}} / J_{0}$ ratio is computed over a range of distances ahead of the tip at each load level, typically $1-2 \leq r /\left(J / \sigma_{0}\right) \leq 4-5$ with the specific values dependent upon the degree of strain hardening. The objective is to sample the stress field at locations outside the finitely deformed $z c n e, r \geq 2 \delta$, but within the process zone applicable for cleavage fracture. Herrens and Read [43]; Miglin, et al. [44] determined fractographically the limit of the cleavage prucess zone as $r=8 \delta$.

Figure 3.7 shows the $J_{\mathrm{FB}} / J_{0}$ ratios computed using this approach for each loading level indicated in Fig. 3.6. The similarity between the SSY and finite-body stress distributions makes the specific $r / \delta$ value used in the calculations unimportant over a wicle range of deformation. In practice, $J_{0}$ calculated by $(3.15)$ is considered valid when the values calculated at $r=3 \delta$ and at $r=8 \delta$ differ by less than 10\%. A larger deviation signals too great a dependence of $J_{0}$ on the critical distance selected and, consequently, a breakdown of the method. Figure 3.8 compares the constraint corrections for fracture toughness computed using the simpler approach with crack-plane stresses given by a $J-Q$ analysis and the more complex approach requiri ${ }^{\sigma}$, computation of stressed volumes within principal stress contours. Differences in the constraint corrections are insignificant for engineering applications..

Table 3.Z̈: Fit coefficients for $\mathrm{SSY}_{\mathrm{Q}=0}$ in (3.16)

\begin{tabular}{|c|c|c|c|c|c|c|c|}
\hline$n$ & $G_{1}$ & $G_{2}$ & $G_{3}$ & $n$ & $G_{1}$ & $G_{2}$ & $G_{3}$ \\
\hline 4.0 & 0.842 & -0.2817 & -0.926 & 10.0 & 1.801 & -0.1169 & -5.169 \\
\hline 5.0 & 1.077 & -0.2312 & -2.181 & 18.0 & 2.219 & -0.0668 & -6.165 \\
\hline 7.0 & 1.422 & -0.1687 & -3.952 & 50.0 & 2.646 & -0.0255 & -6.810 \\
\hline
\end{tabular}




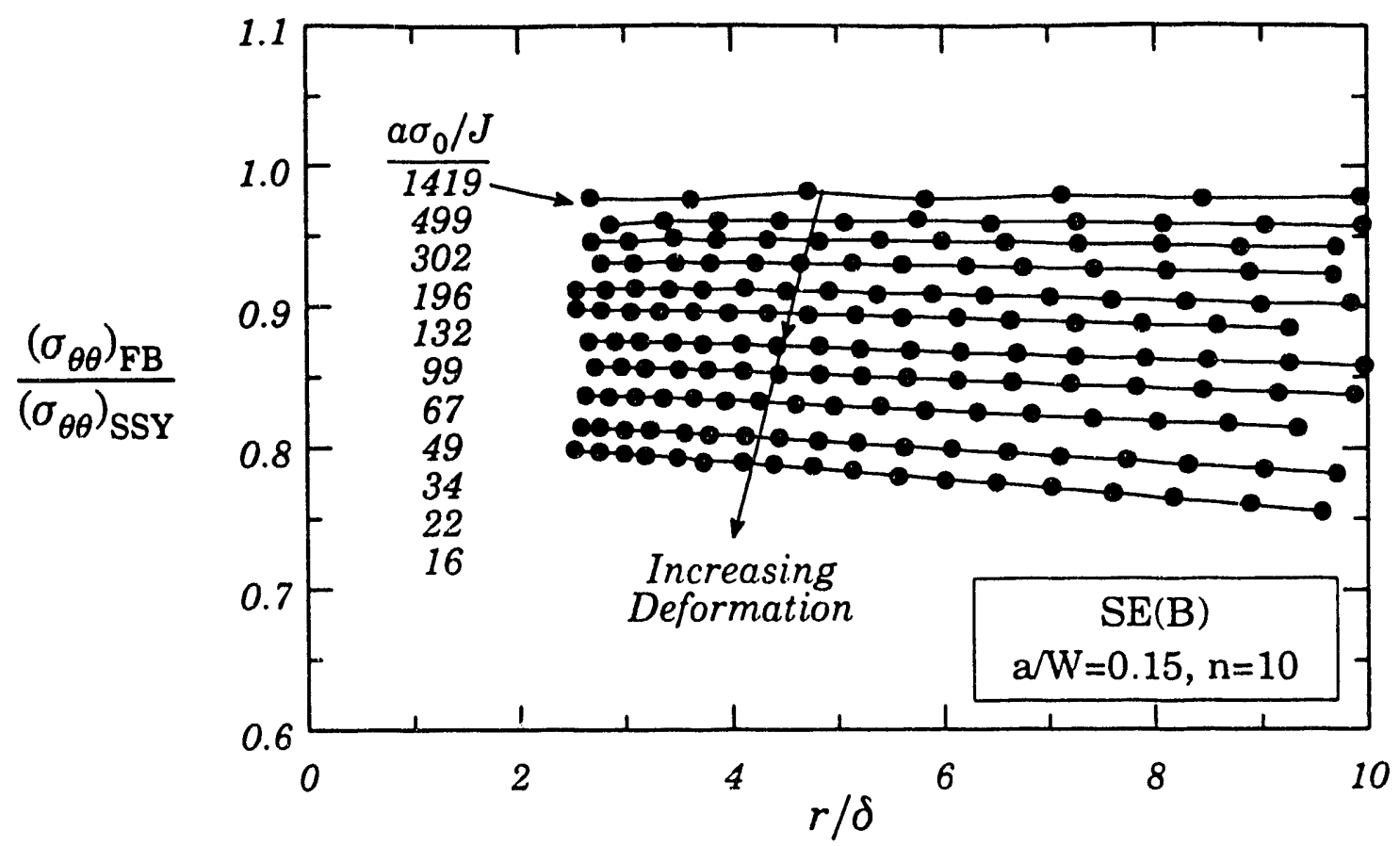

Figure 3.6 Opening stress on the crack plane normalized by $\mathrm{SSY}_{\mathrm{Q}=0}$ at same $J$-applied. $a / W=0.15, n=10 \mathrm{SE}(\mathrm{B})$.

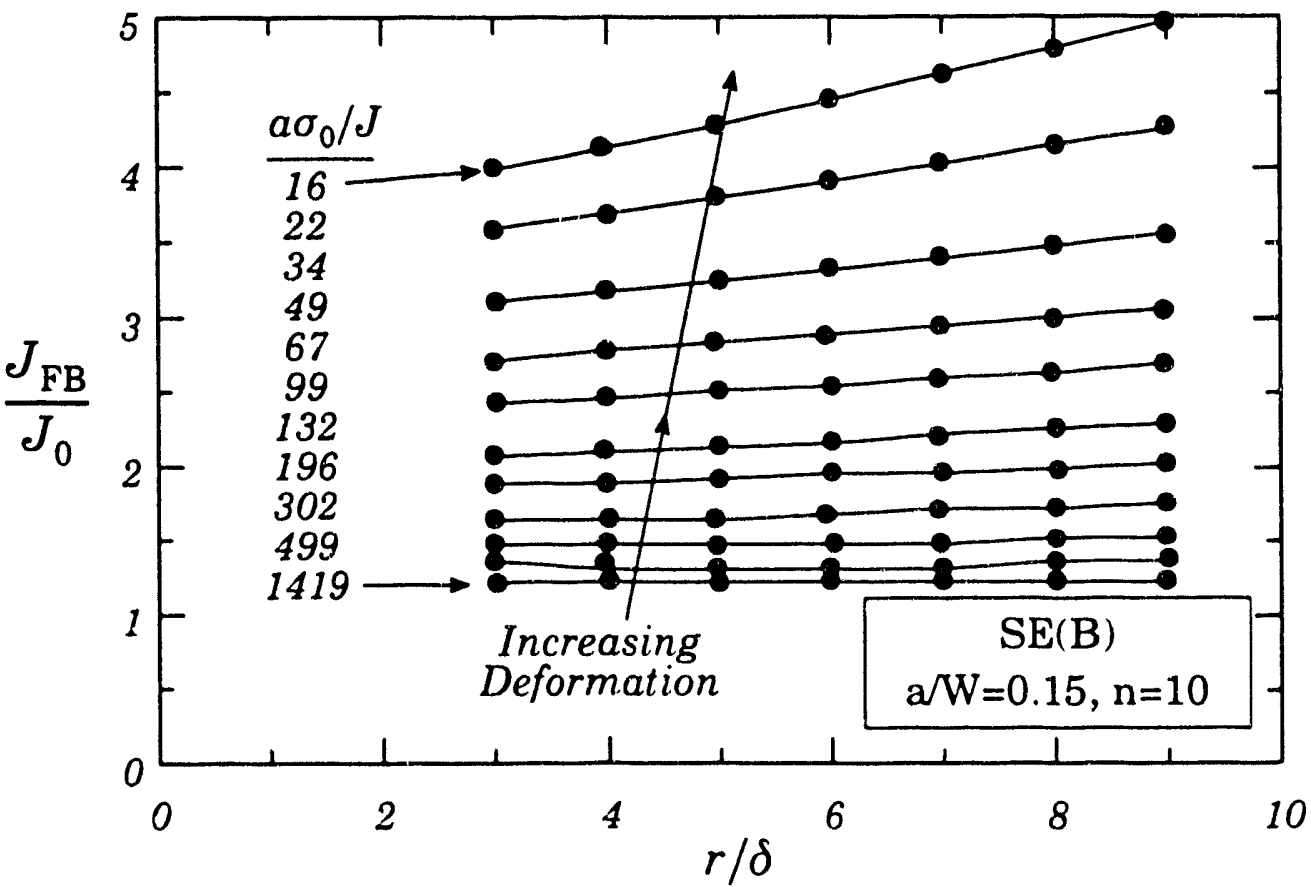

Figure 3.7 Variation of fracture toughness predicted by the micromechanics model using crack-plane stress shown in Fig. 3.6. $a / W=0.15, n=10 \mathrm{SE}(\mathrm{B})$. 


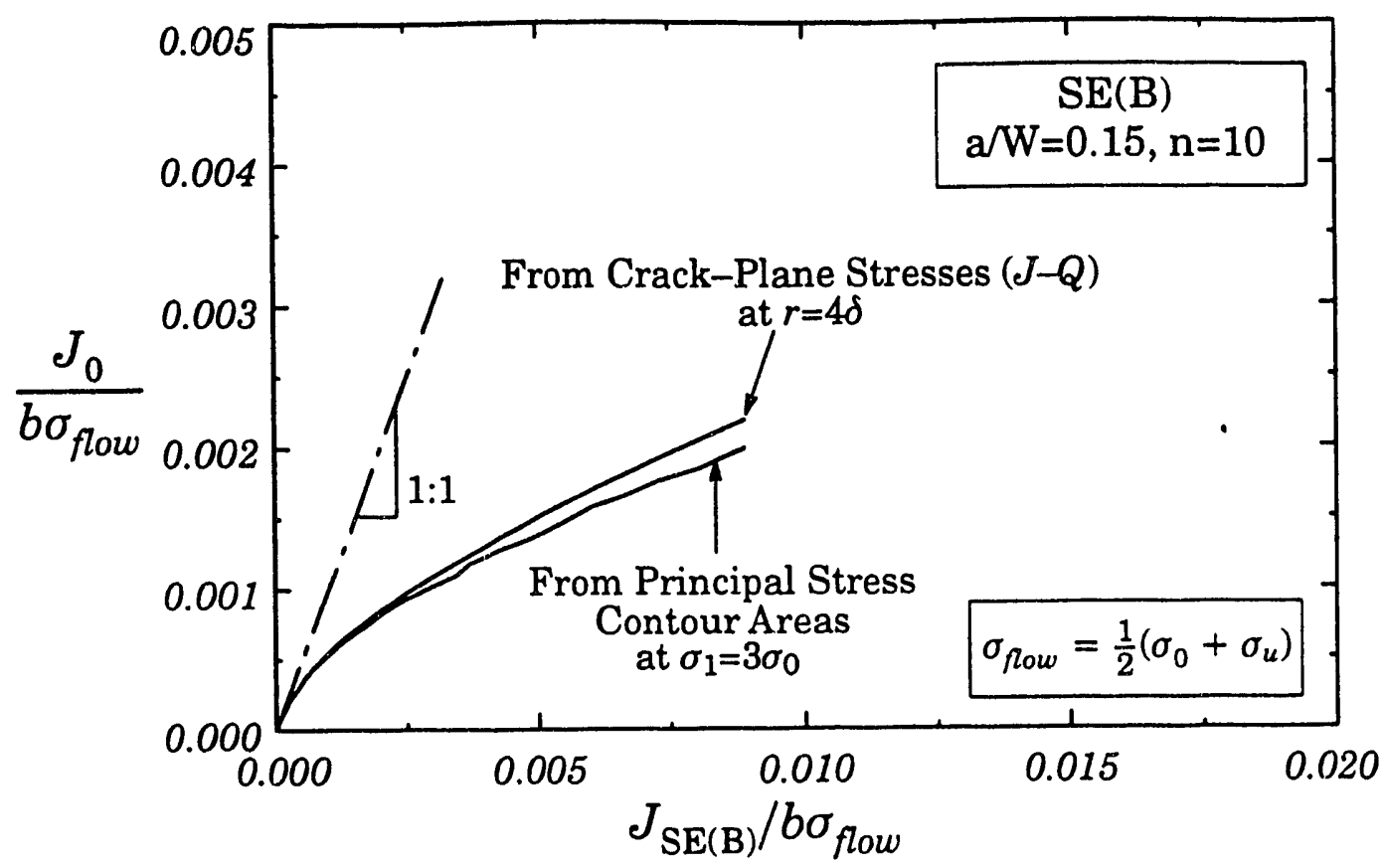

Figure 3.8 Comparison of $J_{0}$ values determined using the crack-plane stress from $J-Q$ and stressed volume techniques for an $a / W=0.15 n=10 \mathrm{SE}(\mathrm{B})$. 


\section{SURFACE CRACKS UNDER BIAXIAL LOADING}

Bass, et. al [45] recently outlined current deficiencies in the understanding of constraint effects on the crack-initiation toughness of shallow surface cracks subjected to uniaxial and biaxial far-field tension loadings. In nuclear applications, the internal pressure alone generates a 1:0.5 biaxial tension loading while the pressurized thermal shock (PTS) event may generate the more severe case of $1: 1$ biaxial tension in addition to a locally severe bending field. The very few testing programs (see Bass, et. al [45] for a review) conducted on biaxially loaded surface cracks report a $25-40 \%$ reduction in toughness values $\left(K_{c}\right)$ relative to the values obtained from $\mathrm{SE}(\mathrm{B})$ and $\mathrm{C}(\mathrm{T})$ specimens containing cracks of similar relative depth. These results imply a significantly increased crack-tip constraint under biaxial loading relative to uniaxial loading. Moreover, the biaxial test results appear to negate the now well established increases in fracture toughness for shallow notch, $S E(B)$ specimens relative to deep notch toughness.

Researchers currently frame discussions of the biaxial $v s$. uniaxial loading influence on constraint in terms of in-plane and out-of-plane effects. Shallow crack SE(B) specimens, for example, exhibit a strong in-plane effect on constraint; the small crack depth relaxes crack-tip stresses when plastic zones sense the nearby free surfaces behind the crack. Outof-plane effects refer to tensile stresses acting parallel to the crack front. While these stresses exist and vary along the front of uniaxially loaded specimens, test programs demonstrate the much smaller influence of thickness $(B)$, which governs the out-of-plane stress, relative to the crack-depth effect once $B$ exceeds a significant fraction of the specimen width (W), usually $B \geq W / 2$. The biaxial test results suggest that mechanically applied, remote out-of-plane stress restores crack-tip triaxiality lost to the shallow-crack in-plane effect. Strength-of-materials type models have been proposed to examine the interaction of in-plane and out-of-plane stresses. Such methods are severely limited since they rely on superposition of stresses which does not apply under elastic-plastic conditions at the crack tip.

The scarcity of testing programs that address biaxial loading effects on fracture toughness and their significant complexity (large plate specimens, scale-model pressure vessels, thermo-mechanical loading, etc.) leave open an experimental resolution of this issue. However, the $J-Q$ and micromechanics concepts described in Sections 2 and 3 provide the analytical framework to clarify the in-plane and out-of-plane effects on crack-tip stress fields (using $J-Q$ ) and on cleavage fracture toughness (using micromechanics).

\subsection{Part-Through Surface Crack Model}

Figure 4.1 shows a flat plate containing a part-through surface crack considered in an initial analytical investigation. The semi-elliptical surface crack has geometric parameters $a / t=0.25,2 c / a=6.0 . \phi=0$ and $\phi=90^{\circ}$ correspond to lines along the free surface and directly ahead of the front at the point of maximum depth. Radial distances ahead of (and normal to) the crack front are denoted $r$. The plate is loaded by a remote uniaxial tension, $\sigma_{z z}^{\infty}$ in one case, and by a remote biaxial tension, $\sigma_{z z}^{\infty}=\sigma_{x x}^{\infty}$ in the second case. The material response is modeled with small-strain, deformation plasticity; the uniaxial flow properties obey a Ramberg-Osgood relationship with hardening exponent $n=10$ and $a=1$.

Symmetry conditions enable consideration of only one-quarter of the full specimen in the finite-element model as shown if Fig. 4.1. The element mesh contains 198020 -node, isoparametric elements and 9800 nodes. The level of mesh refinement in $r$ at each point 


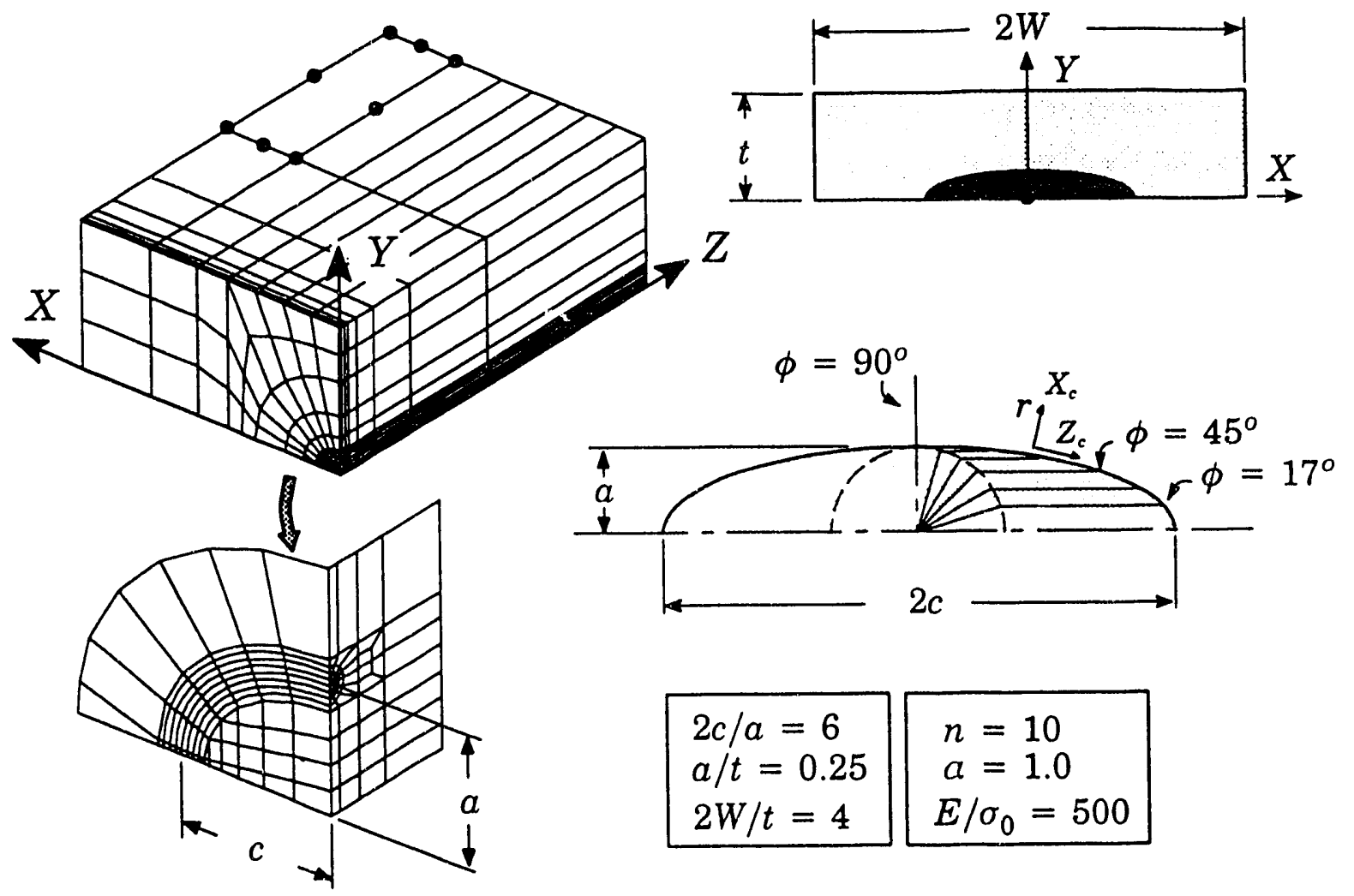

Figure 4.1 Finite-element model for investigation of constraint in surface cracked plate subjected to uniaxial and biaxial remote tension loadings.

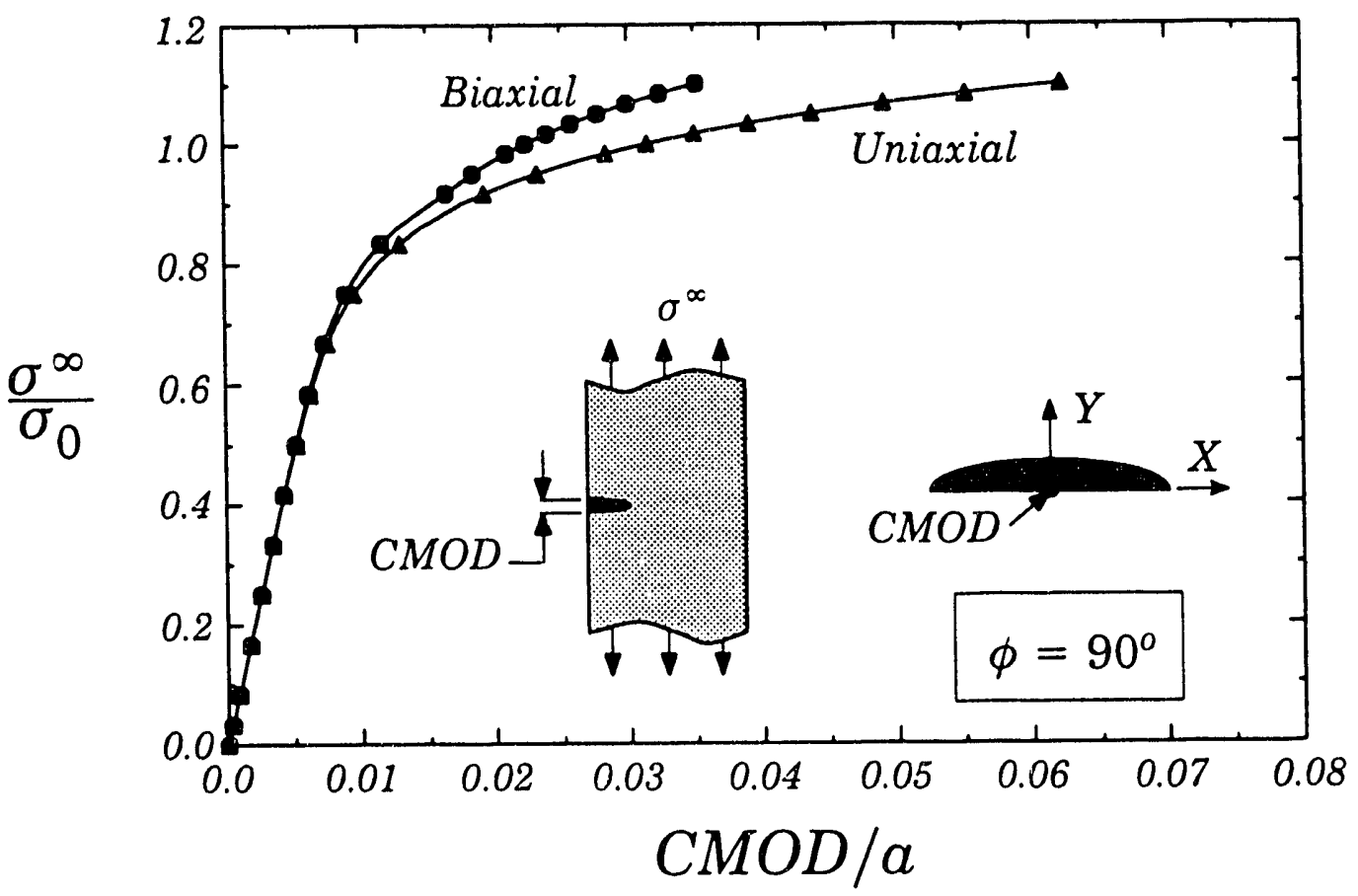

Figure 4.2 Global load-displacement (Crack Mouth Opening Displacement, CMOD) response for surface cracked plate. 


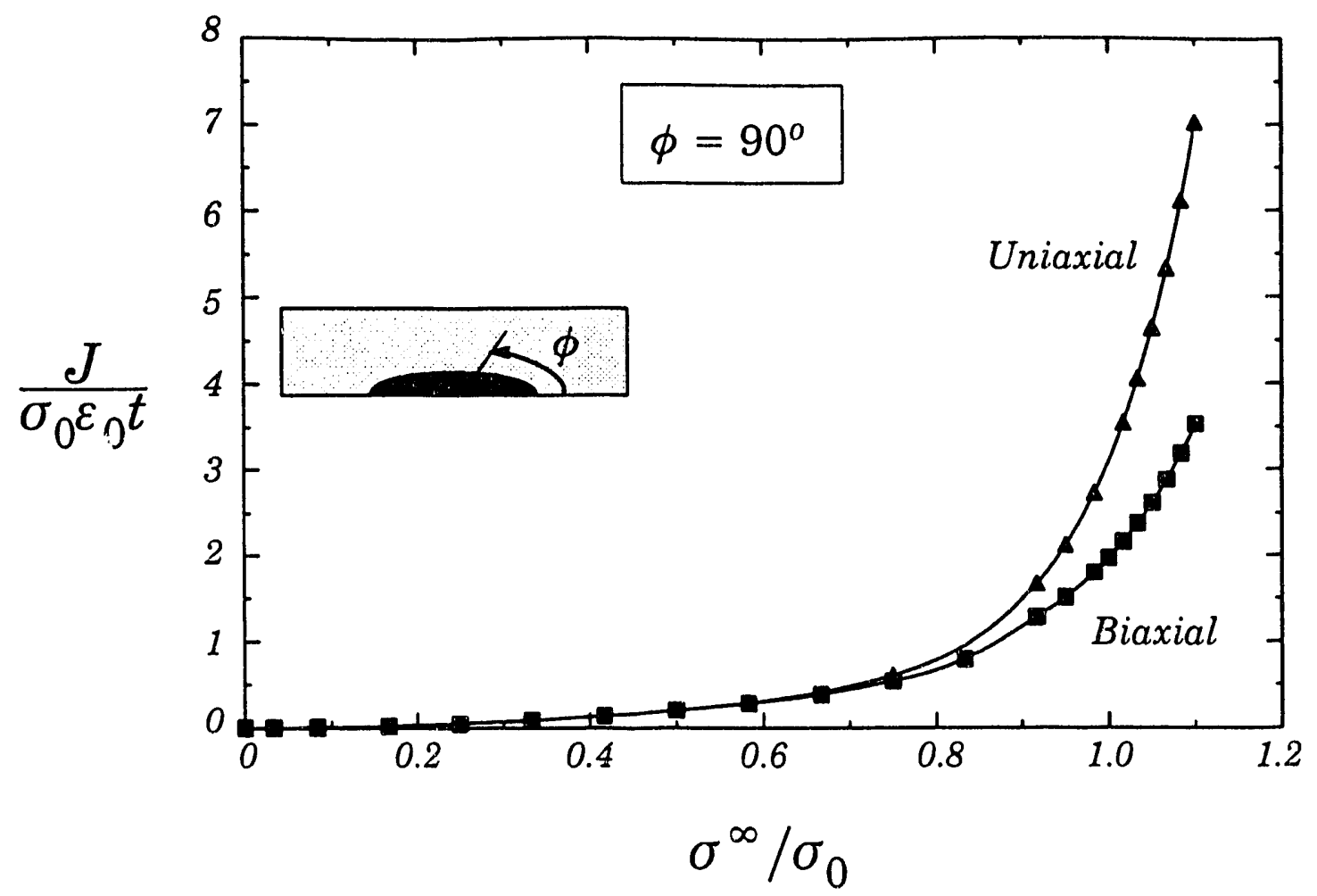

Figure 4.3 $J$-integral values at point of maximum crack depth for uniaxial and biaxial tension loading of surface cracked plate model.

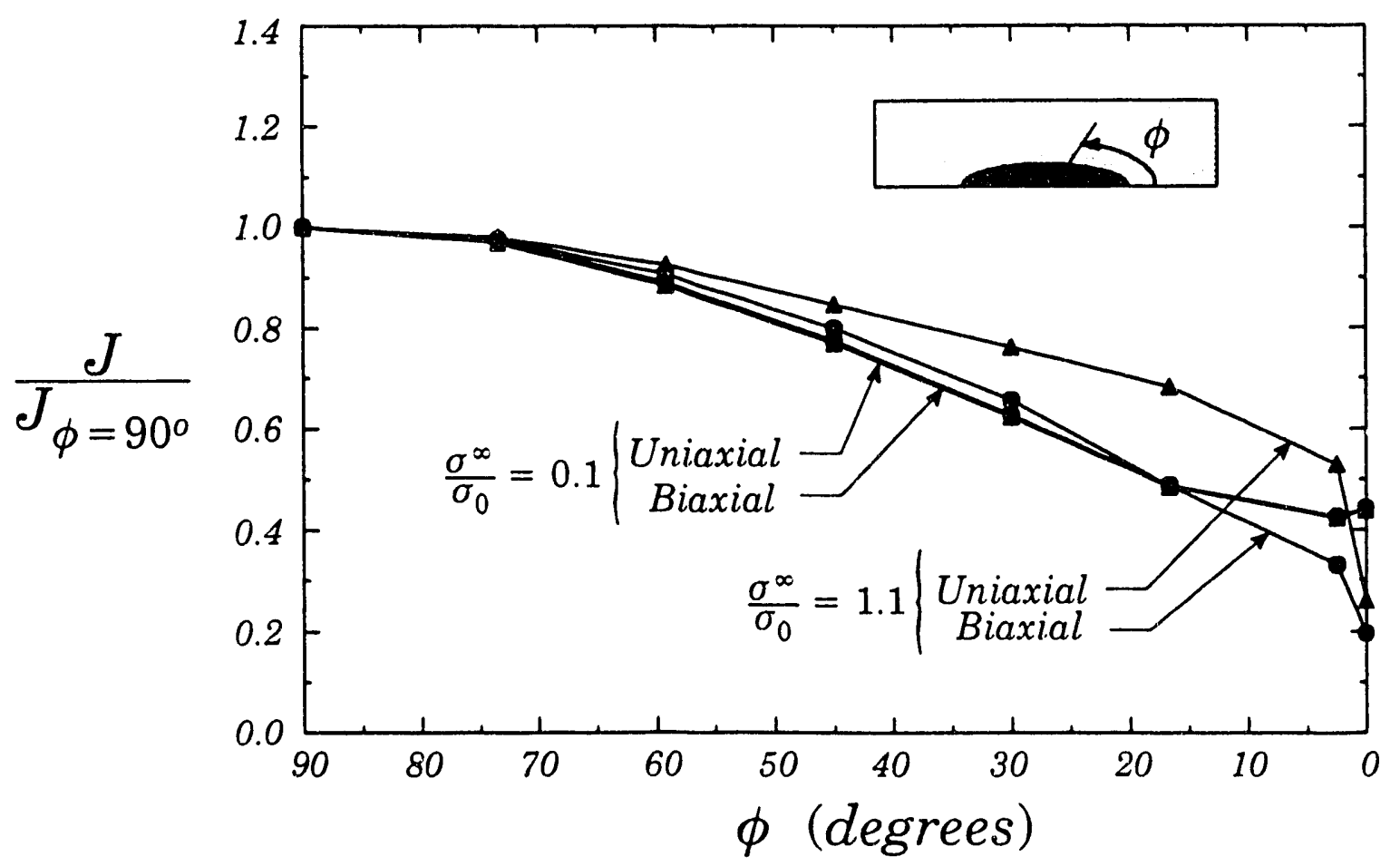

Figure 4.4 $J$-integral along crack front at SSY and LSY load levels normalized by corresponding $J$ at maximum crack depth for each loading level. 
along the crack front matches the refinement employed in previous models [14,31] of twodimensional specimens. The innermost ring of elements incident on the crack front contains degenerate 20-node elements with edge nodes retained in the mid-point location. Initially coincident nodes along the crack front are unconstrained to permit blunting deformations. Uniform reduced integration $(2 \times 2 \times 2)$ in all elements performed satisfactorily in these models.

The intensity of local deformation at each point $s$ along the front is given by [46]

$$
J_{\text {local }}(s)=\lim _{\Gamma_{c} \rightarrow 0} \int_{\Gamma_{c}}\left[W n_{1}-\sigma_{i j} \frac{\partial u_{i}}{\partial_{x_{1}}} n_{j}\right] d \Gamma
$$

where, $W$ denotes the strain-energy density, $\Gamma_{\varepsilon}$ is a vanishingly small contour in the principal normal plane at $s, n$ is a unit normal vector to $\Gamma_{\varepsilon}, \sigma_{i j}$ and $u_{j}$ are Cartesian components of stress in the crack front coordinate system. Numerical evaluation of (4.1) is accomplished with a domain integral method $[46,47]$.

Figure 4.2 shows the overall load-displacement response in terms of Crack Mouth Opening Displacement (CMOD). Under SSY, CMOD remains unaffected by the biaxial loading. With the onset of gross plasticity, however, the biaxial loading provides a significant stiffening effect; at $\sigma^{\infty} / \sigma_{0}=1.1$ the biaxial CMOD is only $55 \%$ of the uniaxial value.

A similar effect of the biaxial loading on the $J$-values can be seen in Fig. 4.3. At the point of maximum crack depth $\left(\phi=90^{\circ}\right)$, the uniaxial $J$-value is twice the biaxial value when both models are loaded to $\sigma^{\infty} / \sigma_{0}=1.1$. The comparison of biaxial and uniaxial distributions for $J_{l o c a l}$ along the crack front is shown in Fig. 4.4. The distributions are identical under SSY but reveal considerable differences under large scale yielding in the region of sharpest front curvature $\left(\phi<30^{\circ}\right)$. The biaxial loading depresses the level of $J$ relative to the uniaxial loading in this region of the crack front.

\subsection{Crack-Front Stress Triaxiality}

Figure 4.5 shows the behavior of near-tip stress triaxiality along radial lines normal to the crack front at $\phi=17^{\circ}$ and $90^{\circ}$ for the uniaxial and biaxial loadings. $Q$-values are defined from opening-mode stresses on the crack-plane using (2.9). At $\phi=90^{\circ}$, the variation of $Q$ with $r$ remains negligible up to the maximum applied load of $\sigma^{\infty} / \sigma_{0}=1.1$. At loadings $\sigma^{\infty} / \sigma_{0} \leq 0.4$, the model lacks sufficient refinement to resolve stresses over the region $2 \leq r /\left(J_{\text {local }} / \sigma_{0}\right) \leq 5$. The crack-tip constraint steadily decreases with increased global loading and plastic deformation ( $Q$ becomes more negative). The biaxial loading exerts only a minor influence toward reducing the constraint loss under large-scale yielding at $\phi=90^{\circ}$.

The $\phi=17^{\circ}$ location on the crack front has high curvature and lies a small distance from the traction-free face of the plate (see Fig. 4.1). Under increased uniaxial loading, $Q$ steadily decreases indicating a gradual loss of stress triaxiality. $Q$ develops a weak dependence on radial distance as the maximum applied loading is approached; $Q$ varies by $\pm 6.7 \%$ from the mean value over $2 \leq r /\left(J_{\text {local }} / \sigma_{0}\right) \leq 5$ at $\sigma^{\infty} / \sigma_{0}=1$.1. The biaxial loading maintains stress triaxiality at significantly higher levels as plastic flow progresses from well-contained through large-scale yielding. The radial dependence increases to $\pm 17 \%$ over $2 \leq r /\left(J_{\text {local }} / \sigma_{0}\right) \leq 5$ at $\sigma^{\infty} / \sigma_{0}=1$.1. The radial dependence of $Q$ when large-scale yielding prevails is induced, in large part, by the nearby free surface.

Alternatively, $Q$ may be computed from values of the mean stress ahead of the crack tip by appealing to the defining equation (2.15). Figure 4.6 illustrates the potential differences 

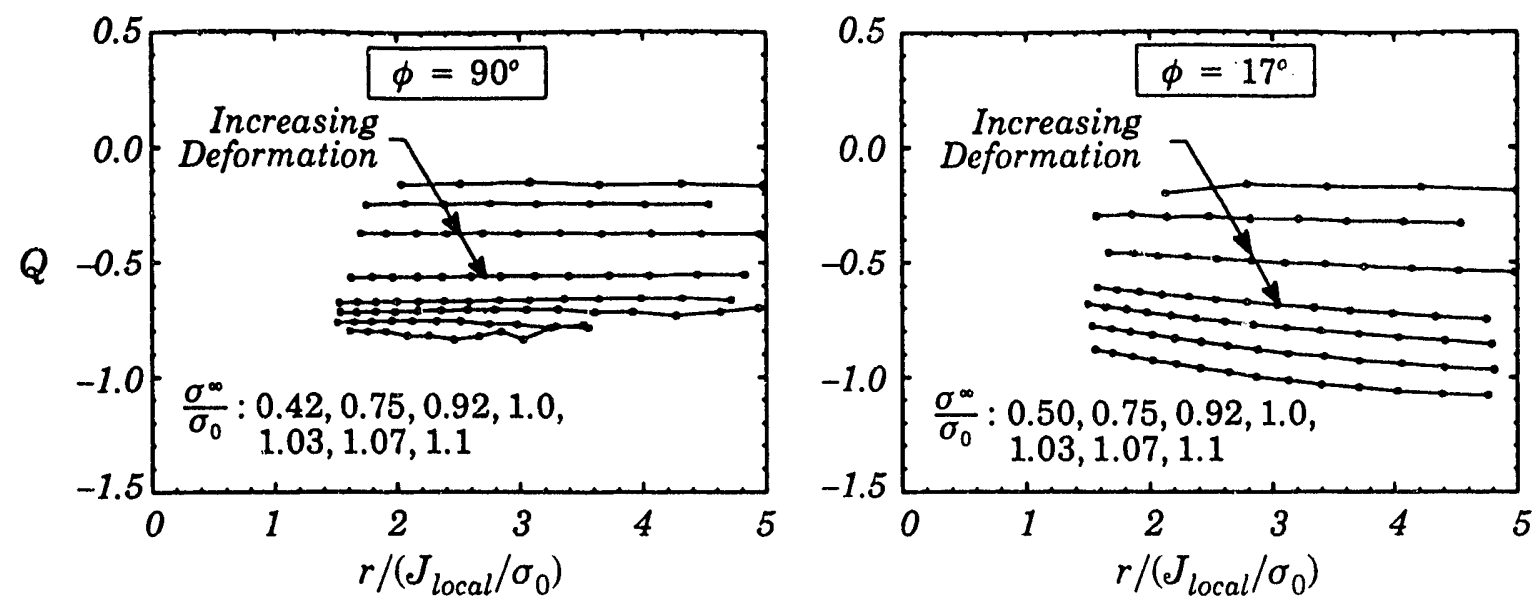

(a) Uniaxial Tension
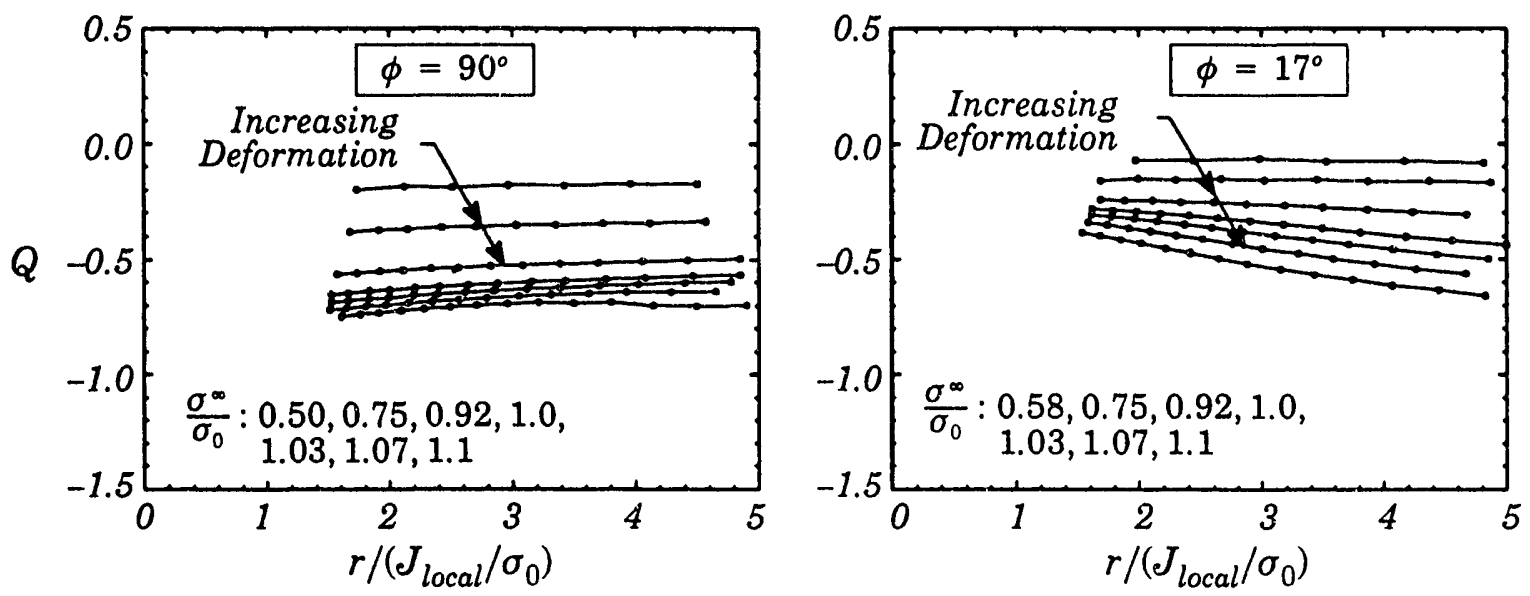

(b) Biaxial Tension

Figure 4.5 Evolution of stress triaxiality in surface cracked plate ahead of crack tip at $\phi=90^{\circ}$ and $17^{\circ}$.

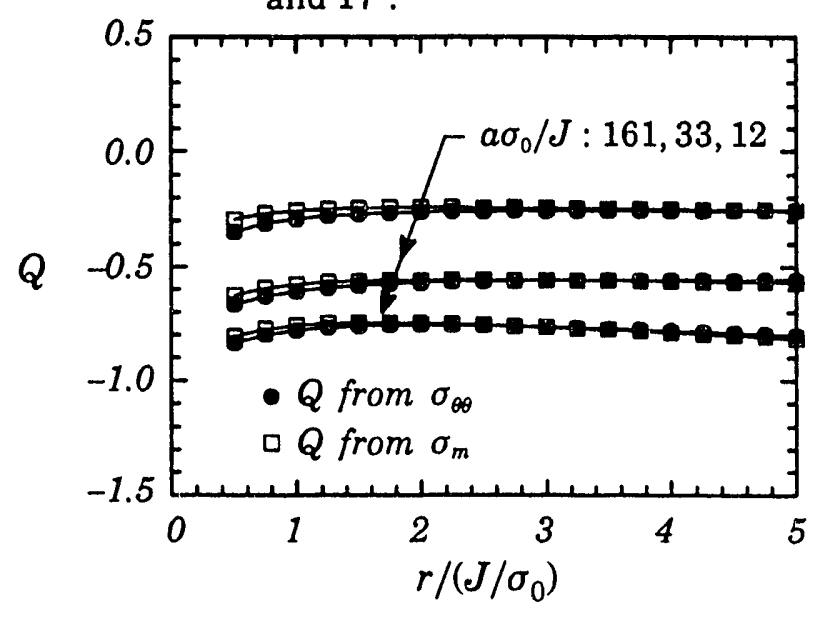

(a) Single-Edge Notched Bend

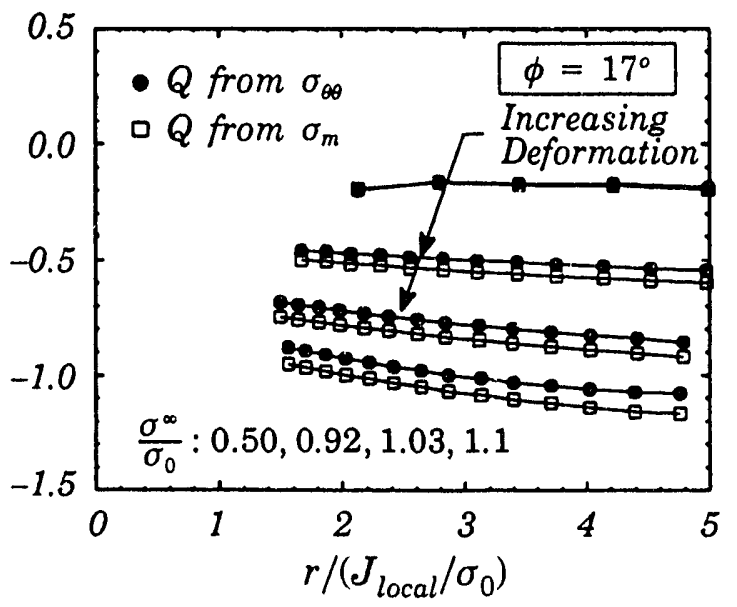

(b) Surface-Cracked Plate

Figure 4.6 Comparison of $Q$-values computed using opening-mode stress (2.9) and the mean stress (2.14): (a) $\operatorname{SE}(B)$ specimen with $a / W=0.15, n=10$; (b) Surfacecracked plate shown in Fig. 4.1 . 
in the two methods to evaluate $Q(2.9,2.15)$. $Q$-values computed from the opening mode stress and the mean stress are shown for a plane-strain model of a shallow notch $\mathrm{SE}(\mathrm{B})$ specimen and for the present surface crack model. The SE(B) specimen exhibits no difference for the two computational procedures while the small differences at high load levels for the surface crack are considered insignificant for engineering applications. The $Q$-values for the surface crack shown in Fig. 4.6.represent the condition (uniaxial vs. biaxial loading, crack-front location) having the largest discrepancy for the two computational procedures. Subsequent discussion of $Q$-values here refer to those defined by (2.9).

Figure 4.7 summarizes the $J-Q$ description of stress triaxiality at different points along the crack front. Both the uniaxial and biaxial cases are taken to the same load level, i.e., $\sigma^{\infty} / \sigma_{0}=1.1 . Q$ is evaluated at $r /\left(J_{l o c a l} / \sigma_{0}\right)=2$ ahead of the crack front. Under uniaxial loading, $Q$ values for $\phi \geq 45^{\circ}$ saturate at -0.8 for large-scale yielding. Near the free surface, $\phi=0$, stress triaxiality is reduced to a level approaching the yield stress even at relatively low loads $(Q \rightarrow-2.0)$. Biaxial loading promotes essentially uniform stress triaxiality, $Q=-0.7$, over much of the crack front. However, at the $\phi=2.4^{\circ}$ and $17^{\circ}$ locations, the influence of biaxial loading is very pronounced. Final $Q$-values for these two locations reveal an increase in stress triaxiality on the order of the yield stress relative to the uniaxial loading response.
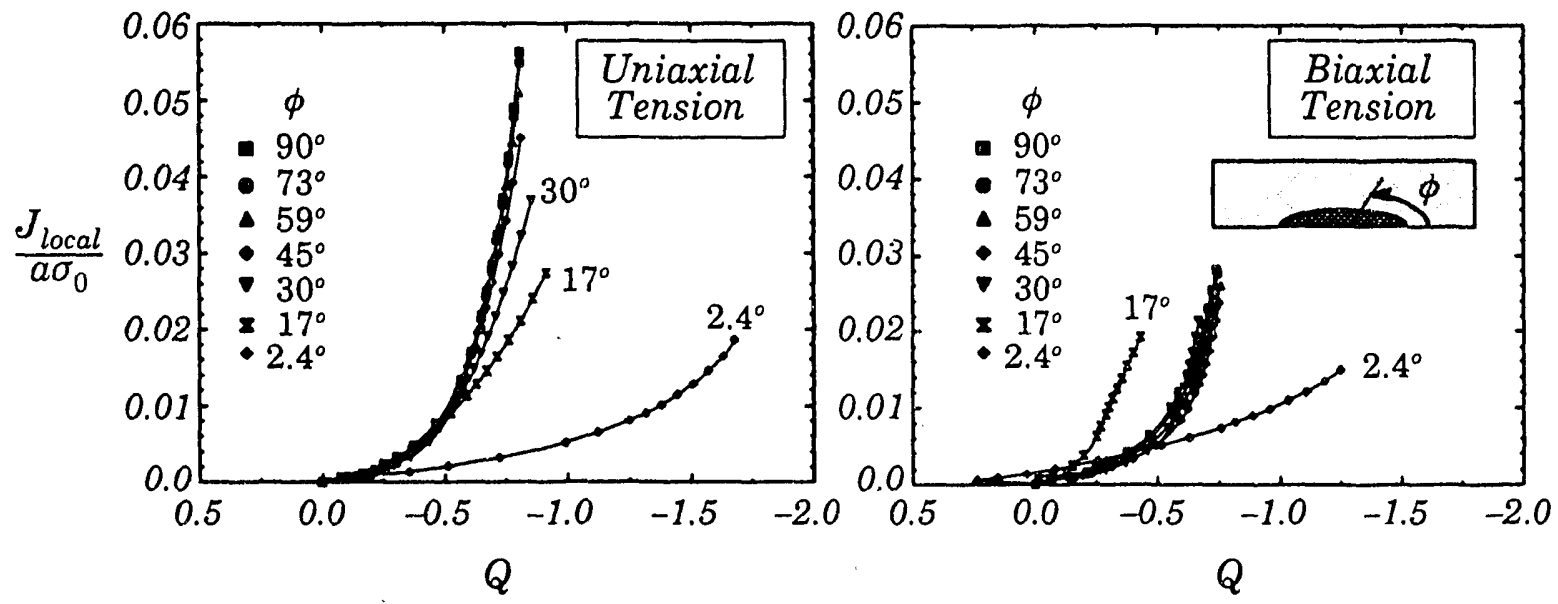

Figure 4.7 Evolution of stress triaxiality in surface-cracked plate with increasing local deformation, as measured by $J_{l o c a l} / a \sigma_{0}$, along radial lines emanating from different points on the crack front. $Q$ is evaluated at $r /\left(J_{\text {local }} / \sigma_{0}\right)=2$. Both geometries are loaded to the same level $\sigma \infty / \sigma_{0}=1.1$.

For crack front locations $\phi \rightarrow 0$, the mechanically imposed biaxial stress, $\sigma_{x x}^{\infty}$, corresponds to a positive $T$-stress (a stress parallel to $X_{c}$, see Fig. 4.1). Under SSY conditions in the surface-cracked plate, the $T$-stress elevates $Q$ slightly above zero in accord with the discussion in Section 2. Under LSY conditions, the $T$-stress brings about a higher level of stress triaxiality near the free surface; however, the $Q$-values are still negative indicating a loss of stress triaxiality relative to the high constraint, reference condition of plane-strain $\mathrm{SSY}_{\mathrm{T}=0}$. Thus, at an identical value of applied- $J$ in uniaxial and biaxial loading, the the crack front region with maximum opening mode stress occurs near $\phi \approx 17^{\circ}$ for the biaxial loading. However, the magnitude of remote loading required to generate the equivalent $J-$ values is larger for the biaxial case (see Fig. 4.3 and 4.4; note the overall larger $J$-values for uniaxial loading). 


\subsection{Matching Structural and Test Specimen Constraint}

Consider the fracture assessment of a structural configuration which is modeled adequately by the surface cracked plate subjected to uniaxial or biaxial loading. The $J-Q$ and micromechanics concepts provide quantitative frameworks to select a corresponding laboratory test specimen, an $\mathrm{SE}(\mathrm{B})$ for example, which produces the same crack front constraint as the structural configuration. The cleavage fracture toughness, $J_{c}$, measured with such a specimen should then be employed in fracture assessments of the structure. These two approaches are illustrated in Figs. 4.8 and 4.9.

Figure 4.8 compares the computed $J-Q$ driving force curves for $\mathrm{SE}(\mathrm{B})$ specimens having a range of $a / \mathrm{W}$ ratios with the driving force curves at $\phi=90^{\circ}$ (uniaxial loading) and $\phi=17^{\circ}$ (biaxial loading) for the surface cracked plate. An $\mathrm{SE}(\mathrm{B})$ specimen with $a / W=0.05$ best matches the evolution of stress triaxiality for the uniaxially loaded plate while an $a / W \approx 0.20$ best matches triaxiality for the biaxial loading. By using the $J-Q$ description of crack front stresses as input to the micromechanics model (as described in Section 3.4), the effects of constraint on cleavage fracture toughness for the surface cracked plate and $\mathrm{SE}(\mathrm{B})$ specimens of selected $a / W$ ratios are predicted as shown in Fig. 4.9. An $\mathrm{SE}(\mathrm{B})$ specimen with $a / W=0.05$ very closely matches the uniaxial loading curve for $\phi=90^{\circ}$ while an $a / W \approx 0.20$ $\mathrm{SE}$ (B) specimen closely matches the biaxial loading curve for $\phi=17^{\circ}$.

Table 4.1 shows predicted values of the remote stress at cleavage fracture determined by the micromechanics approach. Fracture occurs when the applied $J_{0}$ reaches a critical value at the crack front position exhibiting the most stress triaxiality, i.e., the locations at which $J_{\text {local }}$ generates the maximum $J_{0}$ values. As indicated by Figs. 4.7 and 4.9 , critical locations on the crack front are $\phi=17^{\circ}$ for biaxial loading and $\phi=90^{\circ}$ for uniaxial loading. In Table 4.1, three representative values of $J_{0} /\left(a \sigma_{0}\right)$ at fracture are considered. Corresponding values of $J_{l o c a l}$ for the biaxial and uniaxial loadings are found from Fig. 4.9 with the applied loads to produce these $J$-values given by Figs. 4.3 and 4.4. These computations suggest that, despite the higher stress triaxiality of the biaxial loading at $\phi=17^{\circ}$, slightly larger fracture loads arc predicted for the biaxial loading. For this combination of material properties and crack-specimen geometry, the effects of higher stress triaxiality at $\phi=17^{\circ}$ are offset by the lower applied $J$-values at this location on the crack front relative to the $\phi=90^{\circ}$ location.

The potential advantage offered by the micromechanics approach becomes clear from Fig. 4.9. It is not necessary to determine which laboratory specimen matches the structural constraint; rather, any $a / W$ ratio $S E(B)$ can be tested to measure the size independent fracture toughness, $J_{0}$, from which the structural toughness, $J_{c}$, for each loading case (uniaxial and biaxial) is predicted from the corresponding structural response curves shown in Fig. 4.9. When deep notch $\mathrm{SE}(\mathrm{B})$ data is already available, from material qualification tests for example, no additional shallow-crack testing is needed to apply the micromechanics approach. Such applications of the micromechanics model imply that the same fracture mode (cleavage) occurs in both the laboratory specimen and the structural configuration. The current model cannot predict the effects of specimen geometry and loading mode on the fracture toughness $\left(J_{I c}\right)$ which characterizes the initiation of stable, ductile tearing. However, the model does predict when fracture by cleavage becomes highly unlikely. Consider the response for the $a / W=0.5 \mathrm{SE}(\mathrm{B})$ specimen shown in Fig. 4.9 ; if the $J_{0}$ measured with this specimen is sufficiently large, the driving force curve for a shallow notch $\mathrm{SE}$ (B) specimen or for the surface-cracked plate never attain such a large value of $J_{0}$. The model predicts that cleavage, without prior tearing, does not occur, i.e., the interaction of crack-tip plastic 


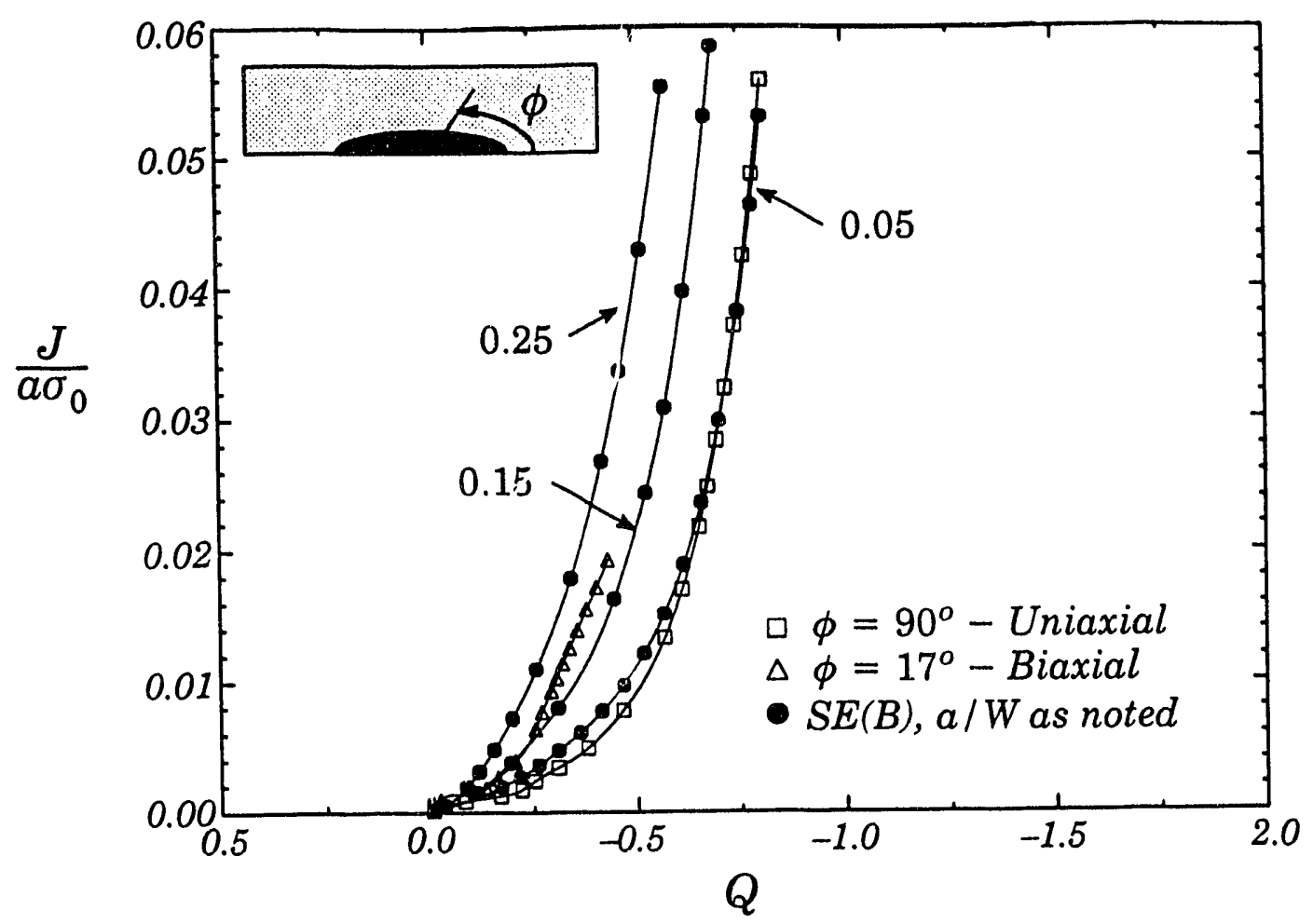

Figure 4.8 Application of $J-Q$ methodology to select $a / W$ ratio in $S E(B)$ specimen for testing that matches stress triaxiality in structural configuration represented by a surface cracked plate loaded in uniaxial or biaxial tension.

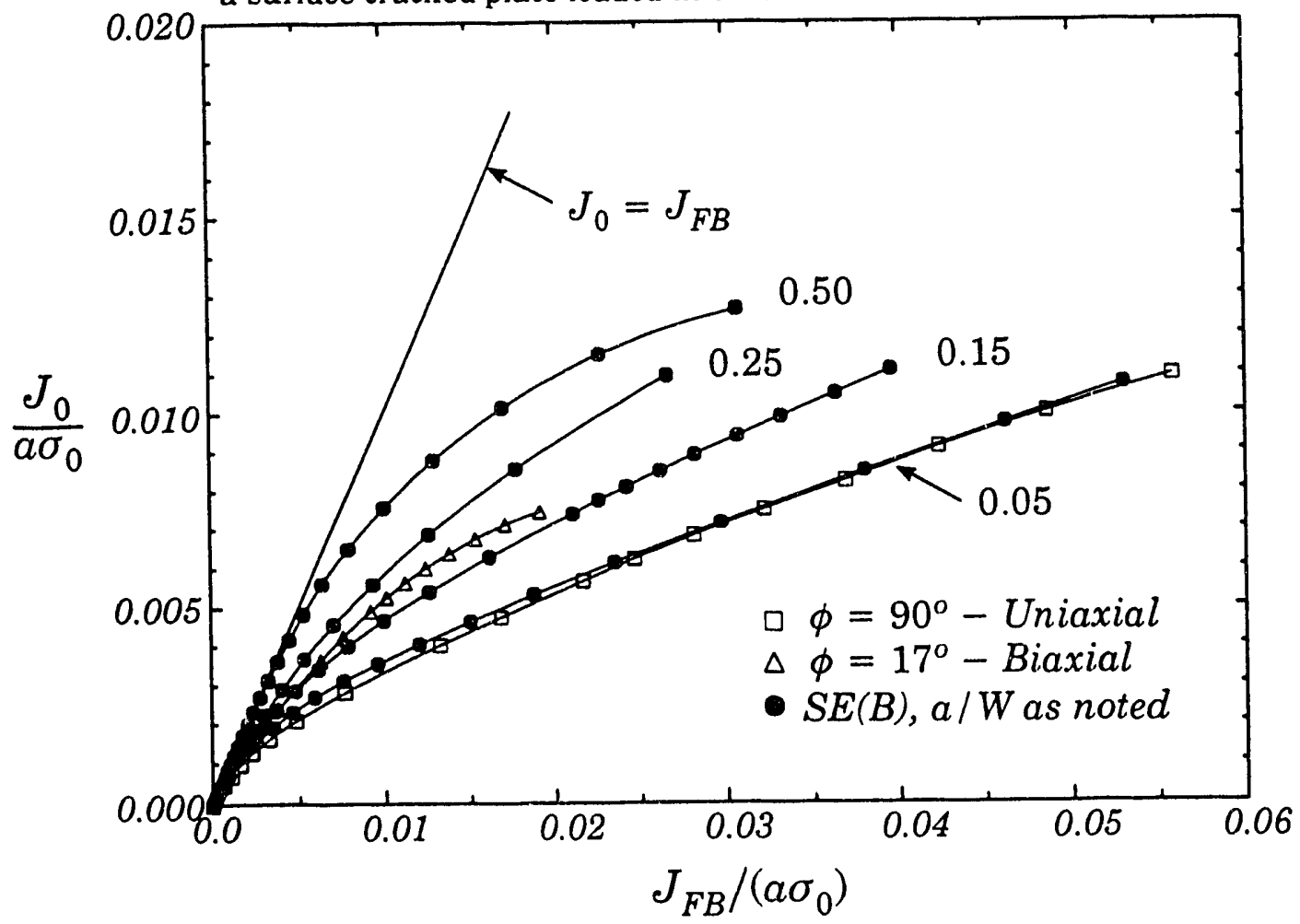

Figure 4.9 Application of micromechanics methodology to select $a / W$ ratio in $\mathrm{SE}(\mathrm{B})$ specimen for testing that matches effective driving force for cleavage fracture in structural configuration represented by a surface cracked plate loading in uniaxial or biaxial tension. 
zones and nearby free surfaces prevents near-tip stresses from achieving the critical levels needed to trigger cleavage.

The SE(B) responses employed in this discussion are obtained from $2-\Gamma$, plane-strain computations. In such cases, constraint matching with a structural configuration is accomplished by varying the absolute specimen size and/or the $a / W$ ratio. For the same $a / W$ ratio, large specimens increase constraint at a given $J$-value relative to small specimens. Similarly, for a fixed specimen size, large $a / W$ ratios increase constraint at a given $J$-value relative to small $a / W$ ratios. Different thicknesses provide yet another means to vary constraint in test specimens, although experimental and computational evidence suggest the thickness effect is much less significant than absolute size or $a / W$ effects when specimens of usual proportions are employed $(B \geqslant W / 2)$.

\begin{tabular}{|c|c|c|c|c|c|c|}
\hline \multicolumn{3}{|c|}{ Table 4.1: Predicted Loads at Fracture for Surface-Cracked Plate } \\
\hline \multirow{2}{*}{$\frac{J_{0}}{a \sigma_{0}}$} & \multicolumn{2}{|c|}{ Biaxial Loading $\left(\phi=17^{\circ}\right)$} & \multicolumn{3}{|c|}{ Uniaxial Loading $\left(\phi=90^{\circ}\right)$} \\
\cline { 2 - 7 } & $\frac{J_{\text {local }}}{a \sigma_{0}}$ & $\frac{J_{\text {local }}}{\sigma_{0} \varepsilon_{0} t}$ & $\frac{\sigma^{\infty}}{\sigma_{0}}$ & $\frac{J_{\text {local }}}{a \sigma_{0}}$ & $\frac{J_{\text {local }}}{\sigma_{0} \varepsilon_{0} t}$ & $\frac{\sigma^{\infty}}{\sigma_{0}}$ \\
\hline 0.00333 & 0.00549 & 0.69 & 0.89 & 0.00960 & 1.20 & 0.86 \\
\hline 0.00665 & 0.01453 & 1.82 & 1.06 & 0.02650 & 3.31 & 1.01 \\
\hline 0.00833 & 0.02300 & 2.88 & 1.14 & 0.03682 & 4.60 & 1.05 \\
\hline
\end{tabular}




\section{CONCLUSIONS}

Our investigations have shown that two-parameters, $J$ and $Q$, suffice to characterize the full range of near-tip environments at the onset of fracture. $J$ sets the size scale of the zone of high stresses and large deformations while $Q$ scales the near-tip stress level relative to a high triaxiality reference stress state. The structure of the $J-Q$ fields has been established by higher-order asypmtotic analysis and full-field numerical calculations within the context of the modified boundary layer formulation. Detailed analyses of finite-width, crack bodies show that the $J-Q$ fields dominate over physically significant size scales, i.e. they represent the environment in which the ductile and brittle mechanisms are operative. Therefore, the $J-Q$ fields furnish the theoretical basis to address onset of clevage fracture, the initiation of ductile tearing, as well as the competition between cleavage fracture and ductile tearing. Indeed, the $J-Q$ theory can provide a framework which allows the cleavage and ductile toughness loci to be measured and utilized in engineering applications.

Constraint effects on cleavage fracture have been the subject of a number of recent studies. The $J-Q$ theory together with a micromechanical model for cleavage predicts that cleavage fracture toughness depends sensitively on near-tip stress triaxiality. The cleavage toughness locus has been measured, for example by Sumpter and Forbes [10] for a mild steel and by Kirk, et al. [42] for A515 steel. The toughness data do show a strong dependence on $Q$. Toughness elevations of about 5 or so have been measured in low constraint crack geometries. Constraint is also expected to exert an influence on the initiation of ductile tearing; however, mechanistic studies of ductile tearing and the limited experimental data suggest that ductile initiation toughness depends less strongly on stress triaxiality. Systematic experimental studies are required to quantify constraint effects on the initiation of ductile tearing.

The experimental determination of a toughness locus can become very costly, requiring considerable material and testing time, especially if toughness data are required for a number of temperatures. An alternative approach for cleavage fracture appears feasible. The constraint correction procedure advocated here uses a limited experimental database to predict cleavage toughness over a broad range of stress triaxiality. The procedure has been applied to several series experiments and the results are very encouraging. The procedure and its theoretical basis are discussed in Section 3. Though it has not been discussed, a similar procedure can be developed (in principle) for the initiation of ductile tearing. As is the case for cleavage fracture toughness, the procedure can be used in conjunction with the $J-Q$ fields to determine geometry- and load-dependent ductile fracture toughness data.

Procedures such as the one in Section 3 hold promise for predicting toughness loci for cleavage and ductile fracture and can facilitate engineering applications of the $J-Q$ approach. However, the incorporation of micromechanics failure criterion in a fracture mechanics methodology is not without its limitations. It is essential that the failure criterion be a sufficiently realistic model of the actual fracture process. In applications where cleavage and ductile failure modes are operative, competing failure processes also must be considered. Unfortunately, the initiation of ductile tearing is also a process involving several competing mechanisms, such as void formation $v s$. void growth and coalescence, void sheet formation and shear localization, and as yet there is no general agreement as to the essential features of a realistic model. Micromechanical models for ductile tearing are necessarily more complex, involving more metallurgical properties for a material, than the models for cleavage fracture. Considering the enormous economic payoffs, however, 
greater efforts are warranted to establish realistic, robust micromechanical models for ductile fracture. 


\section{REFERENCES}

1. Hutchinson, J.W., "Fuidamentals of the Phenomenological Theory of Nonlinear Fracture Mechanics," Journal of Applied Mechanics, Vol. 50, pF. 1042-1051, 1983.

2. Rice, J.R., “A Path Independent Integra! and the Approximate Analysis of Strain Concentration by Notches and Cracks," Journal of Applied Mechanics, Vol. 35, pp. 379-386, 1968.

3. Rice, J.R., and Rosengren, G.F., "Plane Strain Deformation Near a Crack Tip in a Power-Law Hardening Material," Journal of Mechanics and Physics of Solids, Vol. 16, pp. 1-12, 1968.

4. Hutchinson, J.W., "Singular Behavior at the End of a Tensile Crack in a Hardening Material," Journal of Mechanics and Physics of Solids, Vol. 16, pp. 13-31, 1968.

5. McMeeking, R.M., and Parks, D.M., "On Criteria for J-Dominance of Crack-Tip Fields in Large-Scale Yieldirg." Elastic-Plasti; Fracture, ASTM STP 668, J.D. Laandes, J.A. Begley, and G.A. Clark, Ede., American Society for Testing and Materials, Philadelphia, Pennsylvania, pp.175-194, 1:?

6. Shih, C.F. and German, M.D., "Requirements for a One Parameter Characterization of Crack Tip Fields by the HRR Singularity," International Journal of Fracture, Vol. 17, No. 1, pp. 27-43, 1981.

7. Al-Ani, A.M., and Hancock, J.W., "J-Dominance of Short Cracks in Tension and Bending," Journal of Mechanics and Physics of Solids, Vol. 39, pp. 23-43, 1991.

8. Sorem, W.A., Dodds, R.H., and Rolfe, S.T., "Effects of Crack Depth on Elastic Plastic Fracture Toughness," International Journal of Fracture, Vol. 47, pp. '05-126, 1991.

9. Sumpter, J.D.G., " $J_{c}$ Determination for Shallow Notch Welded Bend Specimens," Fatigue and Fracture of Engineering Materials and Structures, Vol. 10, No. 6, pp. 479--493, 1987.

10. Sumpter, J.D.G., and Forbes. A.T., "Constraint Based Analysis of Shallow Cracks in Mild Steel," Proceedings of the International Conference on Shallow Crack Fracture Mechanics Tests and Applications, TWI, Cambridge, England, September 1992.

11. Theiss, T.J., and Bryson, J.R., "Influence of Crack Depth on Fracture Toughness of Reactor Pressure Vessal Steel," Constraint Dffects in Fracture, ASTM STP 1171, E.M. Hackett, K-H. Schwalbe, and R. H. Dodds, Eds., American Society for Testing and Materials, Philadelphia, Pennsylvania, pp. 104-119, 1993.

12. DeCastro, P.M.S.T., Spurrire, J., and Hancock, P., “An Experimental Study of the Crack Length / Specimen Width ( $a$ / W) Ratio Dependence of the Crack Opening Displacement (COD) Test Using Small-Scale Specimens," Fracture Mechanics, ASTM STP 677, C.W. Smith, Ed., Americar Srciety for Testing and Materials, Philadelphia, Pennsylvania pp. 486-497, 1979.

13. O'Dowd, N.P., and Shih, C.F., "Family of Crack-Tip Fields Characterized by a Triaxiality Parameter: Part I - Structure of Fields," Journal of the Mechanics and Physics of Solids, Vol. 29., No. 8, pp. 989-1015, 1991.

14. O'Dowd, N.P., and Shih, C.F., "Family of Crack-Tip Fields Characterized by a Triaxiality Parameter: Part II - Fracture Applications," Journal of the Mechanics and Physics of Solids, Vol. 40. pp. 939-963, 1992.

15. Shih, C. F., O'Dowd, N. P. and Kirk, M. T., "A Framework for Quantifying Crack Tip Constraint," Constraint Effects in Fracture, ASTM STP 1171, E.M. Hackett, K-H. Sch walbe, and R. H. Dodds, Eds., American Society for Testing and Materials, Philadelphia, Pennsylvania, pp. 2-20, 1993.

16. O'Dowd, N. P. and Shih. C. F., "Two-Parameter Fracture Mechanics: Theory and Applications," NUREG/CR-5958, 1993.

17. Li, Y.C. and Wang. T.C., Scientia Sinica (Series A), Vol. 29, pp. 941-955, 1986.

18. Sharma. S.M. and Aravas, N. Journal of Mechanics and Physics of Solids, Vol. 39, pp. 1043-1072, 1991.

19. Xia, L., Wang. T.C. and Shih. C.F., "Higher-Order Analysis of Crack-Tip Fields in Power Law Hardening Materials," to appear in Journal of Mechanics and Physics of Solids. 
20. Ritchie, R.O., and Thompson, A.W., Metallurgical Transactions A, Vol. 16A, pp. 233-248, 1985.

21. Ritchie, R.O., Knott, J.F., and Rice, J.R., "On the Relationship Between Critical Tensile Stress and Fracture Toughness in Mild Steel," Journal of the Mecharics and Physics of Solids, Vol. 21, pp. 395-410, 1973.

22. Rice, J. R. and Johnson, M. A., The Role of Large Crack Tip Georietry Changes in Plane Strain Fracture," in Inelastic Behavior Of Solids, eds. Kanninen et al. McGraw Hill, New York, pp. 641-672, 1970:

23. Needleman A. and Tvergaard, V., "An Analysis of Ductile Rupture Modes at a Crack Tip," Journal of the Mechanics and Physics of Solids, Vol. 35, pp. 151-183, 1987.

24. Betegon, C., and Hancock, J.W., "Two-Parameter Characterization of Elastic-Plastic Crack Tip Fields," Journal of Applied Mechanics, Vol. 58, pp. 104-113, March 1991.

25. Du, Z.-Z., and Hancock, J. W., Journal of Mechanics and Physics of Solids, Vol. 39, pp. 555-567, 1991.

26. Hancock, J.W., Reuter, W.G., and Parks, D.M., "Constraint and Toughness Parameterized by T," Constraint Effects in Fracture, ASTM STP 1171, E.M. Hackett, K.-H. Schwalbe, and R. H. Dodds, Eds., American Society for Testing and Materials, Philadelphia, Pennsylvania, pp. 21-40, 1993.

27. Parks, D.M., "Advances in Characterization of Elastic-Plastic Crack-Tip Fields," to appear in Topics in Fracture and Fatigue, Springer Verlag, 1992.

28. Wang, Y.Y., "On the Two-Farameter Characterization of Elastic-Plastic Crack-Front Fields in Surface-Cracked Plates," Constraint Effects in Fracture, ASTM STP 1171, E.M. Hackett, K.-H. Schwalbe, and R. H. Dodds, Eds., American Society for Testing and Materials, Philadelphia, Pennsylvania, pp. 120-138, 1993.

29. Wang, Y.Y., "A Two-Parameter Characterization of Elastic-Pl 4 stic Crack-Tip and Applications to Cleavage Fracture," Ph.D. Thesis, Department of Mischanical Engineering, MIT, 1991.

30. Kirk, M.T., and Dodds, R.H., "Effect of Weld Metal Strength Mismatch on Elastic-Plastic Fracture Parameters," Civil Engineering Studies, SRS No. 57Q University of Illinois, Urbana, Illinois, August 1992.

31. Dodds, R.H., Anderson, T.L., and Kirk, M.T., "A Framework to Correlate $a / W$ Ratio Effects on Elastic-Plastic Fracture Toughness $\left(J_{c}\right)$," International Journal of Fracture, Vol. 48, pp. 1-22, 1991.

32. Anderson, T.L., and Dodds, R.H., "Specimen Size Requirements for Fracture Toughness' Testing in the Ductile-Brittle Transition Region," Journal of Testing and Evaluation, Vol. 19, pp. 123-134, 1991.

33. Rice, J.R., and Johnson, M.A. in Inelastic Behavior of Solids, (M.F. Kanninen, et al., Eds.), McGraw-Hill, New York, pp. 641-671, 1970.

34. McMeeking, R.M., "Finite Deformation Analysis of Crack-Tip Opening in Elastic-Plastic Materials and Implications for Fracture," Journal of the Mechanics and Physics of Solids, Vol. 25, pp. 357-381, 1977.

35. Williams, M.L., Jounal of Applied Mechanics, Vol. 24, pp. 109-114, 1957.

36. Bilby, B.A., Cardew, G.E., Goldthorpe, M.R. and Howard, I.C., "Size Effects in Fracture," Institution of Mechanical Engineers, London, England, pp. 36-46, 1986.

37. Harlin, G., and Willis, J.R., Proceedings of the Royal Society, Vol. A415, pp. 197-226, 1988.

38. Beremin, F.M, "A Local Criterion for Cleavage Fracture of a Nuclear Pressure Vessel Steel," Metallurgical Transactions, Vol. 14A, pp. 2277-2287, 1983.

39. Wallin, K., Saario, T., and Torronen, K., "Statistical Model for Carbide Induced Brittle Fracture in Steel," Metal Science, Vol. 18, pp. 13-16, 1984.

40. Anderson, T.L., and Stienstra, D., "A Model to Predict the Sources and Misgnitude of Scatter in Toughness Data in the Transition Region," Journal of Testing and Evaluation, Vol. 17, pp. 46-53, 1989. 
41. Wallin, K., "Statistical Aspects of Constraint with Emphasis on Testing and Analysis of Laboratory Specimens in the Transition Region," Constraint Effects in Fracture, ASTM STP 1171, E.M. Hackett, K.-H. Schwalbe, and R. H. Dodds, Eds., American Society for Testing and Materials, Philadelphia, Pennsylvania, pp. 264-288, 1993.

42. Kirk, M.T., Koppenhoeffer, K.C., and Shih, C.F., "Effect of Constraint on Specimen Dimensions Needed to Obtain Structurally Relevant Toughness Measures," Constraint Effects in Fracture, ASTM STP 1171, E.M. Hackett, I. $_{2}-$ H. Schwalbe, and R. H. Dodds, Eds., American Society for Testing and Materials, Philadelphia, Pennsylvania, pp. 79-103, 1993.

43. Herrens, J., and Read, D.T., "Fracture Behavior of a Pressure Vessel Steel in the Ductile-toBrittle Transition Region," NISTIR 88-3099, National Institute for Standards and Technology, Boulder, Colorado, December, 1988.

44. Miglin, M.T., Wade, C.S., and Van Der Sluys, W.A., "Analysis of Fracture Toughness Data for Modified SA508 C12 in the Ductile-to-Brittle Transition Region,"Fracture Mechanics: Twenty-First Symposium, ASTM STP 1074, J.P. Gudas, J.A. Joyce, and E.M. Hackett, Eds., American Society for Testing and Materials, Philadelphia, Pennsylvania, pp. 238-263, 1990.

45. Bass, B.R., Shum, D.K.M, and Walker, J.K., "Constraint Effects on Fractrure Toughness for Circumferentially Oriented Cracks in Reactor Pressure Vessels," NUREG/CR-6008, ORNL/ TM-12131, Oak Ridge National Laboratory, June 1992.

46. Moran, B., and Shih, C.F., "Crack Tip and Associated Domain Integrals from Momentum and Energy Balance," Engineering Fracture Mechanics, Vol. 27, pp. 615-642, 1987.

47. Shih, C.F., Moran, B., and Nakamura, T., "Energy Release Rate Along a Three-Dimensional Crack Front in a. Thermally Stressed Body," International Journal of Fracture, Vol. 30, pp. 79-102, 1986. 

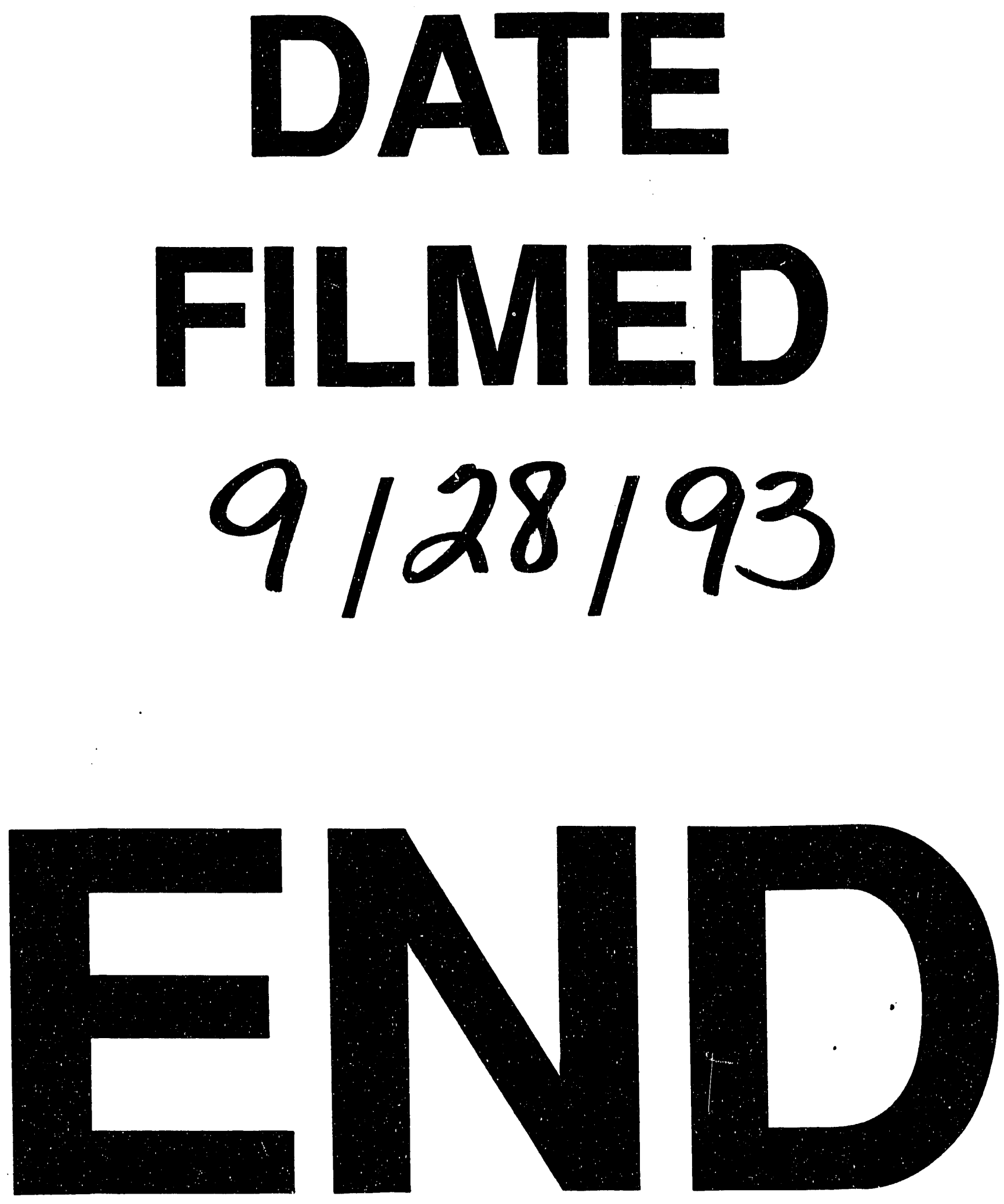
